\title{
Efficient reanalysis techniques for robust topology optimization
}

\author{
Oded Amir, Ole Sigmund, Boyan S. Lazarov and Mattias Schevenels
}

\begin{abstract}
The article focuses on the reduction of the computational effort involved in robust topology optimization procedures. The performance of structures designed by means of topology optimization may be seriously degraded due to fabrication errors. Robust formulations of the optimization problem were shown to yield optimized designs that are tolerant with respect to such manufacturing uncertainties. The main drawback of such procedures is the added computational cost associated with the need to evaluate a set of designs by performing multiple finite element analyses. In this article, we propose efficient robust topology optimization procedures based on reanalysis techniques. The approach is demonstrated on two compliant mechanism design problems where robust design is achieved by employing either a worst case formulation or a stochastic formulation. It is shown that the time spent on finite element analysis within robust topology optimization can be reduced significantly, without affecting the outcome of the optimization process.
\end{abstract}

Keywords Topology optimization, Robust design, Manufacturing errors, Approximate reanalysis

\section{Introduction}

Since it was introduced by Bendsøe and Kikuchi [1988], the material distribution approach to topology optimization has emerged as an effective computational design tool with a wide range of engineering applications (see Bendsøe and Sigmund [2003] for an overview of the theory and applications). One of the main challenges in the development of topology optimization is ensuring the manufacturability of the optimized designs: an optimized design might exhibit poor performance due to errors in fabrication. Examples are MEMS devices manufactured using etching processes, nano-devices manufactured using e-beam lithography or laser micro-machining and macro structures manufactured using milling processes. In these processes, fabrication of a structural detail that is thinner or thicker compared to the optimized design may lead to performance that is far from optimal. Therefore the consideration of manufacturing uncertainties within the optimization process is highly desirable.

Achieving robust designs that are less sensitive to manufacturing errors was the focus of several recent studies. Initially, Sigmund [2009] proposed a robust formulation that considers uniform errors, meaning the fabricated product is uniformly thinner or thicker than the optimized design. Such over-etching and under-etching were simulated using erosion and dilation operators, based on density filtering [Bruns and Tortorelli, 2001; Bourdin, 2001] and projection techniques [Guest et al., 2004; Sigmund, 2007]. The corresponding optimization problem aimed at minimizing the objective functional for the worst of three design cases, namely the intermediate 'blueprint' design (which is to be manufactured), an over-etched (eroded) design and an under-etched (dilated) design. Consequently, good performance was obtained for several magnitudes of uniform errors along the perimeter of the structure. This approach was later modified and improved by Wang et al. [2011], where it was demonstrated that the robust formulation can also ensure local length scale control on both solid and void regions of the intermediate 
design. Recent articles extended the robust formulation to account for random uniform manufacturing errors [Lazarov et al., 2011] as well as for random spatially varying manufacturing errors [Schevenels et al., 2011]. When considering spatial variations, the magnitude of overand under-etching was simulated with the help of random fields defined over the design domain. The objective was to minimize the weighted sum of the mean performance and of the standard deviation of the performance, while both stochastic quantities were approximated by means of either stochastic collocation or a Monte Carlo simulation with a set of 100 random designs. We note that topology optimization with spatially varying geometric uncertainties has also been considered by Chen and Chen [2011] using the level-set method. Furthermore, robust topology optimization with respect to material uncertainties has been addressed by Chen et al. [2010] and recently by Tootkaboni et al. [2012], whereas load uncertainties were discussed by Kogiso et al. [2008] and by Logo et al. [2009]. The focus here is on robust topology optimization procedures; further references and reviews of reliability- and robust-based optimization methods can be found in Beyer and Sendhoff [2007]; Tsompanakis et al. [2008]; Schuller and Jensen [2008].

The main drawback of robust topology optimization procedures is the added computational cost related to the multiple finite element analyses that should be performed within every design cycle. The number of linear systems of equilibrium equations to be solved simultaneously may vary between 3 (for the intermediate, eroded and dilated designs in the worst case approach) to several hundreds or thousands required for a reliable Monte Carlo simulation (in the stochastic approach). As pointed out by Sigmund [2009] and by Wang et al. [2011], the computational burden may be diminished by applying reanalysis techniques that avoid the costly solution of multiple linear systems. The idea behind reanalysis procedures is to re-use a factorization of a certain stiffness matrix in order to efficiently compute an approximation to the solution of another linear system involving a similar stiffness matrix. The effective integration of reanalysis techniques into robust topology optimization procedures is the main concern of this article. The goal is essentially to reproduce the results reported by Wang et al. [2011] and Schevenels et al. [2011] using only a single stiffness matrix factorization per design cycle. In principle, the presented approach can easily be extended to the case of material uncertainties where again a set of similar designs is evaluated simultaneously. However, for demonstrative purposes the focus here is only on optimal design with geometrical variation.

We follow the Combined Approximations (CA) approach for structural reanalysis, originally proposed by Kirsch [1991] for linear static reanalysis. Accurate results and significant savings in computational effort have been reported; for an overview of the method and applications the reader is referred to Kirsch [2008]. The approach has been applied successfully in various classes of structural reanalysis problems, e.g. vibration reanalysis [Kirsch and Bogomolni, 2004], dynamic reanalysis [Kirsch and Bogomolni, 2006] and nonlinear reanalysis [Amir et al., 2008]. In connection with topology optimization, it was shown that CA can be utilized in the reanalysis of truss structures undergoing topological changes, even when the structural model is altered and the number of degrees of freedom is changed [Kirsch and Papalambros, 2001]. More recently, Amir et al. [2009] addressed the integration of approximate reanalysis into standard topology optimization procedures. It was shown that due to the consideration of consistent design sensitivities, even rough approximations of the structural response yield optimized designs that are practically identical to those obtained using standard procedures. Even though the main purpose of the current study is integrating reanalysis into robust formulations, we also offer new insight regarding the utilization of CA in topology optimization in general. Consequently, we believe that the observations presented here lay the foundations for even more effective implementations than those suggested by Amir et al. [2009].

The article is organized as follows. The robust topology optimization problem formulations are reviewed in Section 2. Section 3 is the heart of the article where we present the integration of approximate reanalysis procedures into the robust formulations. In particular, we discuss key aspects in the implementation of $\mathrm{CA}$ and offer important new insight regarding the convergence 
of the reanalysis procedure. This provides a strong basis for an effective application of CA for evaluating several similar designs. Example problems are presented in Section 4 and the accuracy and efficiency of the proposed procedures are evaluated. Finally, the results are discussed and conclusions are drawn in Section 5.

\section{Robust formulations in topology optimization}

The central feature in robust topology optimization is the consideration of a set of designs corresponding to the optimized material distribution, as opposed to the optimization of a single design in standard procedures. The various designs differ in a manner that mimics potential manufacturing errors. In one approach, denoted here as the "worst case" formulation, the set of designs is generated by applying uniform dilation and erosion operations on a certain base design. In another approach, denoted here as the stochastic formulation, random fields are utilized for representing spatial variations in design with respect to the base design. Both approaches and the corresponding optimization problems are briefly reviewed in this section. For a concise presentation, the reader is referred to the articles by Sigmund [2009] and Wang et al. [2011] regarding the worst case formulation and to the article by Schevenels et al. [2011] discussing the stochastic formulation.

In both approaches, the first step consists of generating the base design by applying a density filter [Bruns and Tortorelli, 2001; Bourdin, 2001] on the mathematical design variables field $\boldsymbol{\rho}$. The filtered element density is given by

$$
\widetilde{\rho}_{e}=\frac{\sum_{i \in N_{e}} w\left(\mathbf{x}_{i}\right) v_{i} \rho_{i}}{\sum_{i \in N_{e}} w\left(\mathbf{x}_{i}\right) v_{i}}
$$

where $N_{e}$ is the finite element's neighborhood; $w\left(\mathbf{x}_{i}\right)$ is a weighting function depending on the distance between element $e$ and its neighboring element $i$; and $v_{i}$ is the element's volume. The design sensitivities of a function $f$ with respect to the original densities are computed by the chain rule

$$
\frac{\partial f}{\partial \rho_{e}}=\sum_{i \in N_{e}} \frac{\partial f}{\partial \widetilde{\rho}_{i}} \frac{\partial \widetilde{\rho}_{i}}{\partial \rho_{e}}
$$

with

$$
\frac{\partial \widetilde{\rho}_{i}}{\partial \rho_{e}}=\frac{w\left(\mathbf{x}_{e}\right) v_{e}}{\sum_{j \in N_{i}} w\left(\mathbf{x}_{j}\right) v_{j}}
$$

where $w\left(\mathbf{x}_{e}\right)$ is the weight of element $e$ in the neighborhood of element $i$. In the second step, a parameterized projection function [Xu et al., 2010] is utilized. All filtered densities $\widetilde{\rho}_{i}$ below a threshold value $\eta$ are projected to 0 while all densities above the threshold are projected to 1 . The projection is determined by the function

$$
\overline{\widetilde{\rho}}_{i}=\left\{\begin{array}{cc}
\eta\left[e^{-\beta\left(1-\widetilde{\rho}_{i} / \eta\right)}-\left(1-\widetilde{\rho}_{i} / \eta\right) e^{-\beta}\right] & 0 \leq \widetilde{\rho}_{i} \leq \eta \\
(1-\eta)\left[1-e^{-\beta\left(\widetilde{\rho}_{i}-\eta\right) /(1-\eta)}+\right. & \\
\left.\left(\widetilde{\rho}_{i}-\eta\right) /(1-\eta) e^{-\beta}\right]+\eta & \eta<\widetilde{\rho}_{i} \leq 1
\end{array}\right.
$$

where the parameter $\beta$ governs the curvature of the smooth projection: the higher the value of $\beta$, the closer the function (1) is to the Heaviside step function. In practice, a continuation scheme is applied, raising $\beta$ gradually from 1 to 32 . Another appropriate (and simpler) projection function was proposed by Wang et al. [2011]

$$
\overline{\widetilde{\rho}}_{i}=\frac{\tanh (\beta \eta)+\tanh \left(\beta\left(\widetilde{\rho}_{i}-\eta\right)\right)}{\tanh (\beta \eta)+\tanh (\beta(1-\eta))}
$$


In the worst case approach, the set of designs corresponds to uniform distributions of various predetermined thresholds $\eta$. In particular, we consider the case of three designs: a dilated structure $\left(\eta=\eta_{\min }\right)$, an intermediate structure $\left(\eta=\frac{\eta_{\min }+\eta_{\max }}{2}\right)$ and an eroded structure $(\eta=$ $\left.\eta_{\max }\right)$. In the stochastic approach, a large set of designs is generated according to random spatial distributions of the threshold $\eta$ within a prescribed range, meaning $\eta_{\min } \leq \eta \leq \eta_{\max }$.

\subsection{Worst case formulation}

As a representative design problem we consider the optimization of a compliant force inverter, see Figure 1(a). The objective is to maximize a certain output displacement at the negative global direction. In a standard formulation, the objective function is expressed as $f=\mathbf{l}^{T} \mathbf{u}$ where the vector $\mathbf{l}$ has an entry equal to 1 at the output displacement degree of freedom and zeros otherwise. For achieving a manufacturing-tolerant design, the optimization problem is formulated as a worst case design problem

$$
\begin{array}{cl}
\min _{\boldsymbol{\rho}} & \max \left(\mathbf{l}^{T} \mathbf{u}^{d}, \mathbf{l}^{T} \mathbf{u}^{i}, \mathbf{l}^{T} \mathbf{u}^{e}\right) \\
\text { s.t.: } & \sum_{e=1}^{N} v_{e} \overline{\widetilde{\rho}}_{e}^{d} \leq V_{d}^{\star} \\
& 0 \leq \rho_{e} \leq 1 \quad e=1, \ldots, N \\
\text { with: } & \mathbf{K}\left(\overline{\widetilde{\boldsymbol{\rho}}}^{d}\right) \mathbf{u}^{d}=\mathbf{f} \\
& \mathbf{K}\left(\overline{\widetilde{\boldsymbol{\rho}}}^{i}\right) \mathbf{u}^{i}=\mathbf{f} \\
& \mathbf{K}\left(\overline{\widetilde{\boldsymbol{\rho}}}^{e}\right) \mathbf{u}^{e}=\mathbf{f}
\end{array}
$$

where $\overline{\widetilde{\boldsymbol{\rho}}}^{d}, \overline{\widetilde{\boldsymbol{\rho}}}^{i}$ and $\overline{\widetilde{\boldsymbol{\rho}}}^{e}$ are the dilated, intermediate and eroded material distributions respectively; the corresponding stiffness matrices and displacements are $\left\{\mathbf{K}\left(\overline{\widetilde{\boldsymbol{\rho}}}^{d}\right), \mathbf{K}\left(\overline{\widetilde{\boldsymbol{\rho}}}^{i}\right), \mathbf{K}\left(\overline{\widetilde{\boldsymbol{\rho}}}^{e}\right)\right\}$ and $\left\{\mathbf{u}^{d}, \mathbf{u}^{i}, \mathbf{u}^{e}\right\}$; and $V_{d}^{\star}$ is the maximum allowed volume, imposed as a constraint on the dilated structure. The sensitivity of the function $f=\mathbf{l}^{T} \mathbf{u}$ with respect to a certain projected density distribution is calculated by the adjoint method. First an adjoint system is solved

$$
\mathbf{K}(\overline{\widetilde{\rho}}) \boldsymbol{\lambda}=1
$$

and then the sensitivity with respect to a projected element density is

$$
\frac{\partial f}{\partial \widetilde{\widetilde{\boldsymbol{\rho}}}_{e}}=-\boldsymbol{\lambda}^{T} \frac{\partial \mathbf{K}}{\partial \overline{\widetilde{\boldsymbol{\rho}}}_{e}} \mathbf{u}
$$

This is repeated for all three projections with the appropriate stiffnesses and displacements. Finally, the sensitivity with respect to the original density variables is obtained by the chain rule

$$
\frac{\partial f}{\partial \rho_{e}}=\sum_{i \in N_{e}} \frac{\partial f}{\partial \widetilde{\widetilde{\rho}}_{i}} \frac{\partial \overline{\widetilde{\rho}_{i}}}{\partial \widetilde{\rho}_{i}} \frac{\partial \widetilde{\rho}_{i}}{\partial \rho_{e}}
$$

The same chain rule is applied to compute the sensitivities of the volume constraint with respect to the original density variables.

\subsection{Stochastic formulation}

We focus on the optimization of a gripper mechanism (see Figure 1(b)) as an example design problem to illustrate the stochastic formulation. The stochastic formulation considers spatially varying manufacturing errors [Schevenels et al., 2011]. The projection threshold is modeled as a random field $\eta(\mathbf{x}, \theta)$, where $\mathbf{x}$ is the position in space and $\theta$ is the coordinate in the random dimension [Doob, 1953; Kolmogorov, 1956]. 


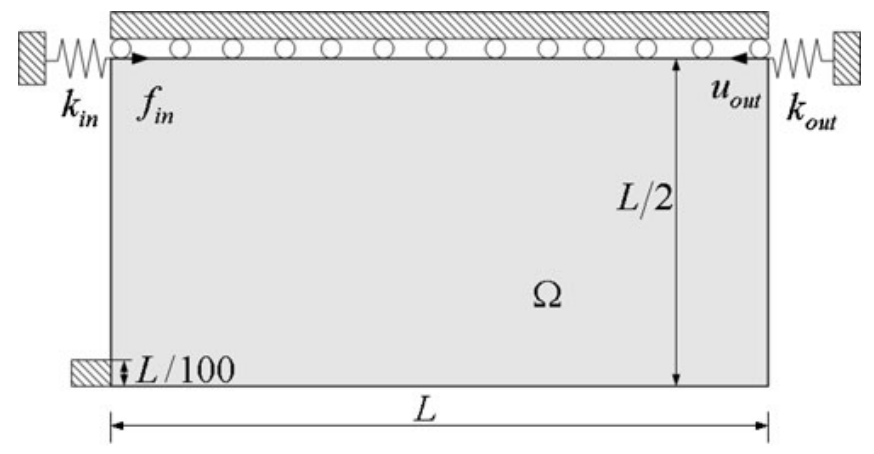

(a) Compliant force inverter [Wang et al., 2011].

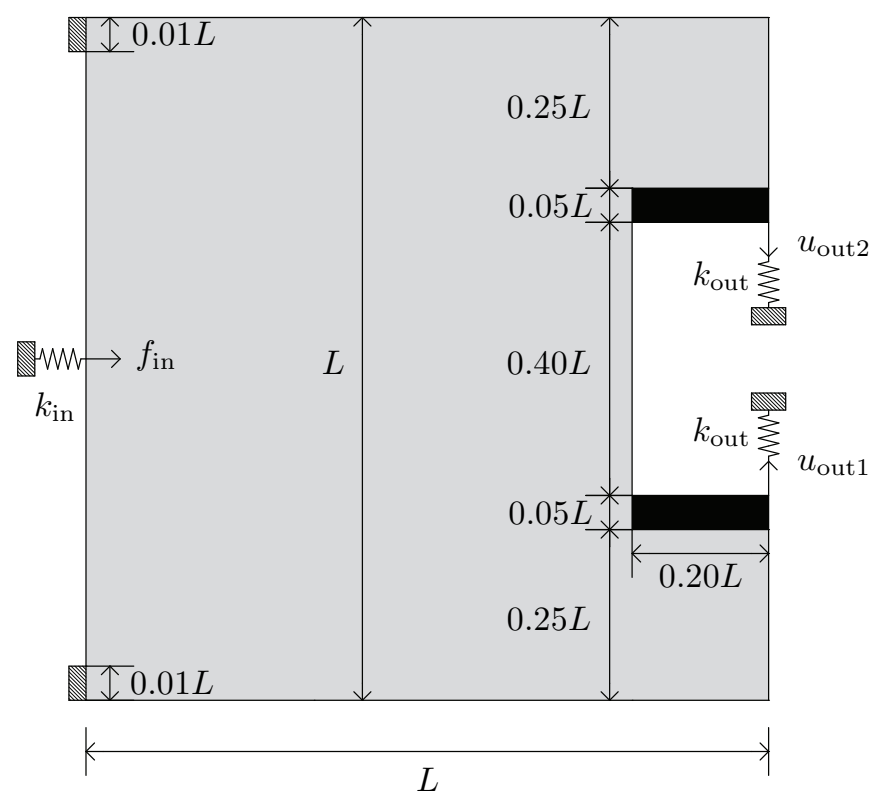

(b) Gripper mechanism [Schevenels et al., 2011].

Figure 1: Design domain, boundary conditions and loads for the two example problems. 
A detailed description of the procedure to simulate the random field $\eta(\mathbf{x}, \theta)$ can be found in [Schevenels et al., 2011]. Essentially, a two-step procedure is followed. First, a realization of a Gaussian field $\zeta(\mathbf{x}, \theta)$ is generated as follows:

$$
\zeta(\mathbf{x}, \theta)=\sum_{k=1}^{M} \xi_{k}(\theta) \phi_{k}(\mathbf{x})
$$

i.e. the random field is expressed as a linear combination of deterministic basis functions $\phi_{k}(\mathbf{x})$ with random coefficients $\xi_{k}(\theta)$. The basis functions $\phi_{k}(\mathbf{x})$ depend on the field's covariance function and can be determined by means of the Karhunen-Loève method [Ghanem and Spanos, 1991] or the Expansion Optimal Linear Estimation (EOLE) method [Li and Der Kiureghian, 1993]. A squared exponential covariance function with a correlation length $l_{\mathrm{c}}=0.3 L$ is assumed here, where $L$ is the size of the design domain (see Figure 1(b)). The random coefficients $\xi_{k}(\theta)$ in equation (7) are independent standard Gaussian variables; they can be simulated by means of a random number generator.

Second, the following transformation is applied:

$$
\eta(\mathbf{x}, \theta)=\gamma(\zeta(\mathbf{x}, \theta))
$$

where $\gamma$ is a nonlinear mapping that translates a realization of the Gaussian field $\zeta(\mathbf{x}, \theta)$ to a realization of a field with the target marginal probability distribution. A uniform marginal probability distribution between 0.4 and 0.6 is used in the present study.

The random character of the projection threshold $\eta(\mathbf{x}, \theta)$ is controlled entirely by the random variables $\xi_{k}(\theta)$. The element densities obtained by projecting the filtered design variables using the random projection threshold $\eta(\mathbf{x}, \theta)$ can therefore be denoted as $\overline{\tilde{\boldsymbol{\rho}}}(\boldsymbol{\rho}, \boldsymbol{\xi})$, where $\boldsymbol{\xi}$ is a vector collecting the random variables $\xi_{k}(\theta)$. Figure 2 shows eight realizations of the element densities $\overline{\tilde{\boldsymbol{\rho}}}(\boldsymbol{\rho}, \boldsymbol{\xi})$ obtained for eight different realizations of the projection threshold $\eta(\mathbf{x}, \theta)$. The effect of an erosion/dilation manufacturing error varying in space can be observed.

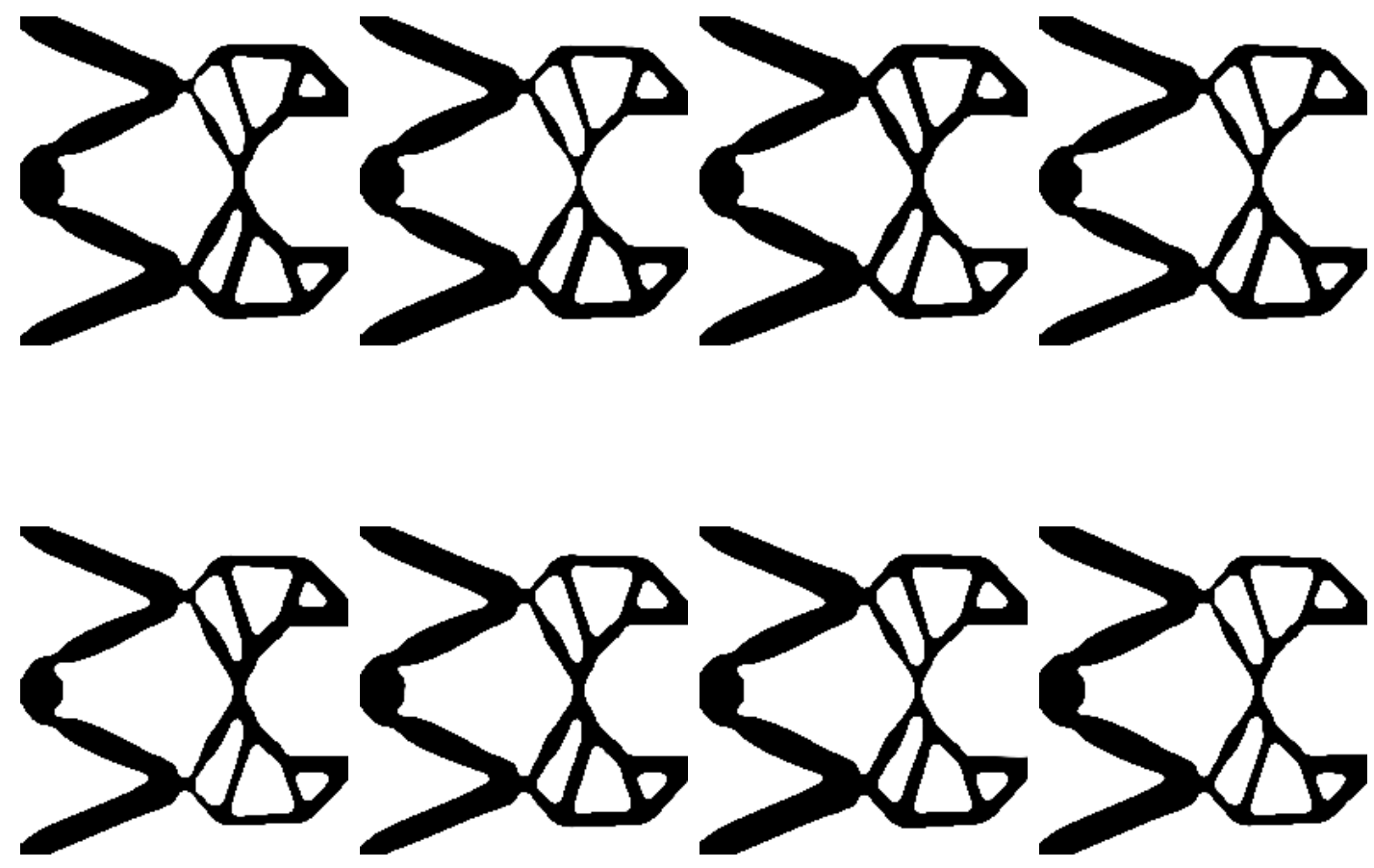

Figure 2: Examples of realizations of spatially varying manufacturing errors in compliant gripper fabrication, generated using a random field.

The objective of the gripper design problem is to maximize the gripping displacements $u_{\text {out } 1}$ and $u_{\text {out } 2}$, meaning minimize $f=u_{\text {out } 2}-u_{\text {out } 1}$. In a standard formulation, this objective function 
is again expressed as $f=\mathbf{l}^{T} \mathbf{u}$ where the vector $\mathbf{l}$ has the entries -1 and 1 at the "out1" and "out2" degrees of freedom respectively, and zeros otherwise. In the robust formulation, the aim is to minimize a weighted sum of the mean performance and the standard deviation of the performance

$$
\begin{array}{cl}
\min _{\boldsymbol{\rho}} & m_{f}(\boldsymbol{\rho})+w \sigma_{f}(\boldsymbol{\rho}) \\
\text { s.t.: } & m_{V}(\boldsymbol{\rho}) \leq V^{\star} \\
& 0 \leq \rho_{e} \leq 1 \quad e=1, \ldots, N \\
\text { with: } & \mathbf{K}(\boldsymbol{\rho}, \boldsymbol{\xi}) \mathbf{u}(\boldsymbol{\rho}, \boldsymbol{\xi})=\mathbf{f}
\end{array}
$$

where $m_{f}(\boldsymbol{\rho})$ and $\sigma_{f}(\boldsymbol{\rho})$ are the mean value and the standard deviation of the performance $f=\mathbf{l}^{T} \mathbf{u}$

$$
\begin{aligned}
m_{f}(\boldsymbol{\rho}) & =\mathbb{E}\{f(\boldsymbol{\rho}, \boldsymbol{\xi}(\theta))\} \\
\sigma_{f}(\boldsymbol{\rho}) & =\sqrt{\mathbb{E}\left\{[f(\boldsymbol{\rho}, \boldsymbol{\xi}(\theta))]^{2}\right\}-[\mathbb{E}\{f(\boldsymbol{\rho}, \boldsymbol{\xi}(\theta))\}]^{2}}
\end{aligned}
$$

The weighting factor $w$ is set to 1 in the present study. For each random design, the volume is computed based on the projected densities

$$
V=\sum_{e=1}^{N} v_{e} \overline{\widetilde{\rho}}_{e}
$$

Then the volume constraint is imposed on the mean value of all the random designs

$$
m_{V}(\boldsymbol{\rho})=\mathbb{E}\{V(\boldsymbol{\rho}, \boldsymbol{\xi}(\theta))\}
$$

The mean volume is constrained to be lower than or equal to a prescribed value $V^{\star}$. The derivatives of the objective and constraints with respect to a certain design variable $\rho_{e}$ are obtained by differentiation of the expressions for the mean and standard deviation

$$
\begin{aligned}
\frac{\partial m_{f}}{\partial \rho_{e}} & =\mathbb{E}\left\{\frac{\partial f}{\partial \rho_{e}}\right\} \\
\frac{\partial \sigma_{f}}{\partial \rho_{e}} & =\frac{1}{\sigma_{f}}\left(\mathbb{E}\left\{f \frac{\partial f}{\partial \rho_{e}}\right\}-m_{f} \frac{\partial m_{f}}{\partial \rho_{e}}\right) \\
\frac{\partial m_{V}}{\partial \rho_{e}} & =\mathbb{E}\left\{\frac{\partial V}{\partial \rho_{e}}\right\}
\end{aligned}
$$

The derivatives $\frac{\partial f}{\partial \rho_{e}}$ are computed by the adjoint method for every random projected density distribution and then translated to the design variables space using the chain rule, just like in Eqs. (4), (5) and (6). The derivatives $\frac{\partial V}{\partial \rho_{e}}$ for each of the designs are obtained by differentiating (9) and applying the chain rule (6).

\section{A reanalysis approach to robust formulations in topology op- timization}

For the purpose of obtaining an approximate response of numerous similar designs considered at each optimization cycle, we apply Kirsch's Combined Approximations (CA) approach for structural reanalysis [Kirsch, 1991, 2008]. Originally, CA was introduced as a reduced basis method, where the basis vectors correspond to a truncated series expansion. Later, Kirsch et al. [2002] showed that CA can also be seen as a particular case of the Preconditioned Conjugate 
Gradient (PCG) method. In this section, we first review the formulation of CA and demonstrate the advantages of implementing it as a PCG rather than in the original manner. Then, we present a consistent sensitivity analysis procedure that accounts for an approximation in the solution of the analysis equations. This is followed by a study focusing on the convergence of the reanalysis procedure, offering new insight which is crucial for effectively applying CA when multiple structures are to be evaluated simultaneously.

\subsection{Kirsch's Combined Approximations as a PCG procedure}

In every topology optimization design cycle, the linear system of finite element equilibrium equations to be solved is

$$
\mathbf{K u}=\mathbf{f}
$$

where $\mathbf{K}$ is the stiffness matrix, $\mathbf{u}$ is the unknown displacements vector and $\mathbf{f}$ is the external forces vector. Instead of solving the full system of equations, it is possible to efficiently find an approximation $\widetilde{\mathbf{u}}$ that will be sufficiently accurate for the purpose of optimization

$$
\begin{aligned}
\widetilde{\mathbf{u}} & \approx \mathbf{u} \\
\mathbf{K} \widetilde{\mathbf{u}} & \approx \mathbf{f}
\end{aligned}
$$

In the Combined Approximations approach, the displacements are first expressed as an infinite series

$$
\mathbf{u}=\left(\mathbf{I}-\mathbf{K}_{0}^{-1} \Delta \mathbf{K}+\left(-\mathbf{K}_{0}^{-1} \Delta \mathbf{K}\right)^{2}+\left(-\mathbf{K}_{0}^{-1} \Delta \mathbf{K}\right)^{3}+\ldots\right) \mathbf{u}_{1}
$$

where $\mathbf{K}_{0}$ is a "reference" stiffness matrix given in its factorized form, $\Delta \mathbf{K}=\mathbf{K}-\mathbf{K}_{0}$ and $\mathbf{K}_{0} \mathbf{u}_{1}=\mathbf{f}$. The main feature of CA is the utilization of only a few series terms from (11) as basis vectors in a reduced basis solution. Considering only the first $s$ series terms, the approximation of the displacements is expressed as

$$
\widetilde{\mathbf{u}}=y_{1} \mathbf{u}_{1}+y_{2} \mathbf{u}_{2}+\ldots+y_{s} \mathbf{u}_{s}=\mathbf{R}_{B} \mathbf{y}
$$

where $\mathbf{R}_{B}$ is an $n \times s$ matrix containing the basis vectors $\mathbf{u}_{1}, \mathbf{u}_{2}, \ldots, \mathbf{u}_{s}$ and $\mathbf{y}$ is a vector of $s$ unknowns. Replacing $\mathbf{u}$ in (10) with $\widetilde{\mathbf{u}}$ and premultiplying both sides by $\mathbf{R}_{B}^{T}$ gives the reduced system of equations

$$
\mathbf{R}_{B}^{T} \mathbf{K} \mathbf{R}_{B} \mathbf{y}=\mathbf{R}_{B}^{T} \mathbf{f}
$$

In practical implementation, the basis vectors are generated sequentially using the relation

$$
\mathbf{K}_{0} \mathbf{u}_{i}=-\Delta \mathbf{K} \mathbf{u}_{i-1}
$$

This requires one matrix-vector multiplication and one triangular solve using the factors of $\mathbf{K}_{0}$. For computing the reduced stiffness matrix, $s$ additional matrix-vector multiplications are needed. In many cases it is beneficial to orthonormalize the basis vectors using a Gram-Schmidt procedure, because the reduced matrix $\mathbf{R}_{B}^{T} \mathbf{K} \mathbf{R}_{B}$ tends to become ill-conditioned when more than only a few basis vectors are used. In the context of CA procedures, this was originally proposed by Leu and Huang [1998]. The accuracy of the approximation is then improved when a rather large basis is utilized, but for the extra cost of orthogonalization.

It was shown [Kirsch et al., 2002] that the CA procedure is mathematically equivalent to a PCG procedure where the factors of $\mathbf{K}_{0}$ serve as preconditioners. Assuming positive definiteness of both $\mathbf{K}_{0}$ and $\mathbf{K}$, the Cholesky factorization $\mathbf{U}_{0}^{T} \mathbf{U}_{0}=\mathbf{K}_{0}$ is used to precondition the system of equations (10). In other words, the iterative Conjugate Gradient method [Hestenes and Stiefel, $1952]$ is applied to solve the preconditioned system

$$
\left(\mathbf{U}_{0}^{-T} \mathbf{K} \mathbf{U}_{0}^{-1}\right)\left(\mathbf{U}_{0} \mathbf{u}\right)=\mathbf{U}_{0}^{-T} \mathbf{f}
$$


The equivalence of CA and PCG enables to re-cast the reanalysis problem as a PCG procedure, aimed at computing an approximation $\widetilde{\mathbf{u}}$ using only a small number of PCG iterations. In the context of topology optimization, such a procedure was recently implemented by Amir and Sigmund [2011], using only one matrix factorization for the whole design process. One advantage of re-casting the reanalysis problem as a PCG is the computational efficiency: each PCG iteration requires one matrix-vector multiplication and one triangular solve, so it is slightly cheaper than the original CA procedures. Another advantage lies in the superior numerical robustness of PCG, which is demonstrated in the following example.

Comparing numerical robustness: standard CA approach vs. PCG We consider the compliance minimization of the well-known MBB-beam, see for example Bendsøe and Sigmund [2003]. The focus is on the structural reanalysis corresponding to the 10th design cycle. The FE mesh consists of $60 \times 20$ square bi-linear elements; the allowed volume fraction is $50 \%$; the SIMP penalty factor is 3.0; and the filter radius is 1.5 . The reference stiffness matrix given in factorized form corresponds to an entirely solid domain, as in Amir and Sigmund [2011]. The approximation $\widetilde{\mathbf{u}}$ corresponding to the previous design cycle is used as an initial guess for the current reanalysis.

The approximation $\widetilde{\mathbf{u}}$ is computed using four different schemes: 1) Standard CA; 2) CA with an orthonormal basis; 3) PCG with the factors of the reference matrix as preconditioners; 4) CA with an orthonormal basis, and assuming exact orthonormality. The difference between schemes 2 ) and 4) is that if exact orthonormality is achieved, then a simple expression can be used for computing the contribution of each added basis vector. Otherwise a small system of equations in the form of (12) should be solved every time another basis vector is added. In the CA procedures, we use up to 30 basis vectors which are equivalent to 30 PCG iterations. For each number of PCG iterations / basis size, we evaluate the accuracy of the approximation by measuring the relative norm of residual forces $\frac{\|\mathbf{f}-\mathbf{K} \widetilde{\mathbf{u}}\|_{2}}{\|\mathbf{f}\|_{2}}$. The results are depicted in Figure 3. It can be seen, that with up to 10 PCG iterations / basis vectors, the approximations computed using the four schemes are exactly the same. Then, the accuracy of the standard CA procedure deteriorates due to ill-conditioning of the reduced stiffness matrix. The orthonormal basis yields results that are close to those obtained by PCG, but it should be mentioned that the orthogonalization is not precise once the size of the basis exceeds 16 basis vectors, presumably due to round-off errors. For this reason, the solution assuming orthonormality diverges from this point. It is therefore concluded that it is beneficial to re-cast Kirsch's Combined Approximations approach as a PCG. This provides superior computational robustness, as well as improved efficiency and ease of implementation. Accordingly, throughout the remainder of this article we refer mainly to the PCG formulation of the reanalysis problem, which is also the one we implemented for the purpose of this study.

\subsection{Sensitivity analysis for approximate reanalysis}

In conventional topology optimization procedures following the nested approach, it is assumed that the analysis equations are solved accurately. Then, the adjoint method is utilized to compute design sensitivities efficiently, for the objective functional and for each of the constraints. When approximate reanalysis is performed, the analysis equations are not necessarily solved accurately so the standard adjoint approach is not applicable in general. In order to overcome this difficulty, it is necessary either to accept a certain error in the design sensitivities or to perform the sensitivity analysis in a manner that is consistent with approximate reanalysis.

This study addresses the robust design of compliant mechanisms. The objective is to minimize a certain performance measure which is related directly to the displacements. As described above, this is typically expressed as $f=\mathbf{l}^{T} \mathbf{u}$, where the vector $\mathbf{l}$ consists of the values 1 or -1 at the relevant degrees of freedom and zeros otherwise. In standard procedures, the adjoint 


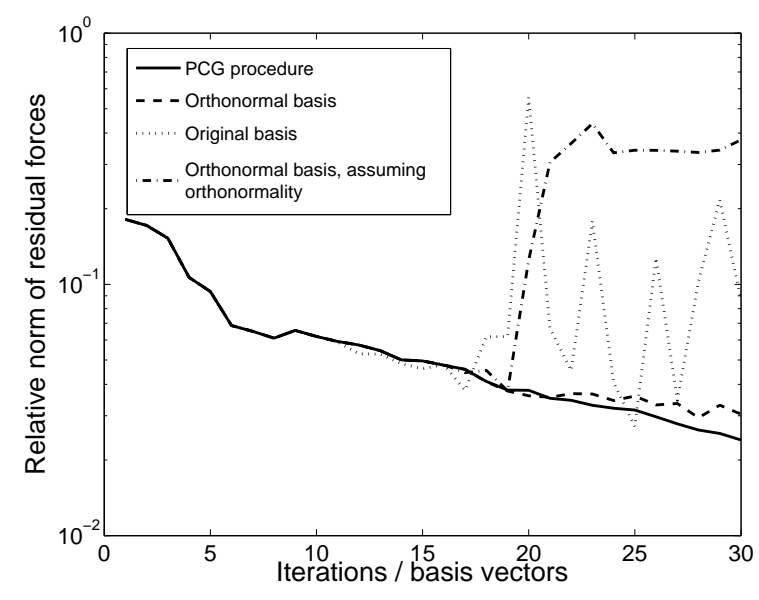

Figure 3: Comparing the accuracy of four implementations of the reanalysis procedure.

equation system to be solved is

$$
\mathbf{K} \boldsymbol{\lambda}=\mathbf{l}
$$

and then the sensitivity of the objective with respect to an element density is

$$
\frac{\partial f}{\partial \rho_{e}}=-\boldsymbol{\lambda}^{T} \frac{\partial \mathbf{K}}{\partial \rho_{e}} \mathbf{u}
$$

Approximate sensitivity analysis One possible approach is to view also the adjoint equation system (13) as a reanalysis problem. This means that for each design to be evaluated, we solve two reanalysis problems simultaneously. In the PCG framework this is conveniently performed using a block-PCG procedure [O'Leary, 1980]. Two approximations are obtained for every design: $\widetilde{\mathbf{u}}$ and $\widetilde{\boldsymbol{\lambda}}$ corresponding to the displacements and to the adjoint variables. The error associated with these approximations is ignored and approximate design sensitivities are computed by

$$
\frac{\partial f}{\partial \rho_{e}}=-\widetilde{\boldsymbol{\lambda}}^{T} \frac{\partial \mathbf{K}}{\partial \rho_{e}} \widetilde{\mathbf{u}}
$$

This approach led to very good results in previous studies [Amir et al., 2010; Amir and Sigmund, 2011] and is also applicable for the robust formulation, as shown in Section 4. However, it lacks mathematical consistency and may fail when aiming to reduce the number of PCG iterations. In particular, it is observed that the errors in the solution of the adjoint system (in terms of relative residual forces) are larger than the errors corresponding to the solution of the structural analysis equations, by one to two orders of magnitude. This is due to the ground structure's aspect ratio and to the location of loads and supports, and was also observed in the study by Amir et al. [2010].

Consistent sensitivity analysis A more rigorous approach involves the derivation of design sensitivities that are consistent with the approximation resulting from the reanalysis procedure. In the context of utilizing Kirsch's Combined Approximations in topology optimization, consistent sensitivity analysis was first introduced by Amir et al. [2009]. The approach was later generalized in Amir et al. [2010] for approximations obtained by forcing early termination of PCG when it is applied as a standard iterative equation solver. The main idea is that when applying the adjoint method for sensitivity analysis, the complete iterative procedure performed in order to obtain the approximation is taken into account. Then the design sensitivities are accurate and consistent with respect to the approximate representation of the problem. 
For a clear presentation of the consistent adjoint procedure, we first review the PCG procedure applied. Aiming to solve the linear system $\mathbf{K u}=\mathbf{f}$ with the preconditioner $\mathbf{K}_{0}=\mathbf{U}_{0}^{T} \mathbf{U}_{0}$, the procedure can be outlined as follows

1. Set the initial guess $\mathbf{u}_{1}$.

2. Compute the initial residual $\mathbf{r}_{1}$ and direction vector $\mathbf{p}_{1}: \mathbf{r}_{1}=\mathbf{f}-\mathbf{K u}_{1}, \mathbf{z}_{1}=\mathbf{K}_{0}^{-1} \mathbf{r}_{1}$, $\mathbf{p}_{1}=\mathbf{z}_{1}$.

3. For $i=1:$ maxiter do
(a) $\alpha_{i}=\frac{\mathbf{r}_{i}^{T} \mathbf{z}_{i}}{\left(\mathbf{K} \mathbf{p}_{i}\right)^{T} \mathbf{p}_{i}}$
(b) $\mathbf{u}_{i+1}=\mathbf{u}_{i}+\alpha_{i} \mathbf{p}_{i}$
(c) $\mathbf{r}_{i+1}=\mathbf{r}_{i}-\alpha_{i} \mathbf{K} \mathbf{p}_{i}$
(d) If $\left\|\mathbf{r}_{i+1}\right\|_{2}<\epsilon\|\mathbf{f}\|_{2}$ break.
(e) $\mathbf{z}_{i+1}=\mathbf{K}_{0}^{-1} \mathbf{r}_{i+1}$.
(f) $\beta_{i}=\frac{\mathbf{r}_{i+1}^{T} \mathbf{z}_{i+1}}{\mathbf{r}_{i}^{T} \mathbf{z}_{i}}$
(g) $\mathbf{p}_{i+1}=\mathbf{z}_{i+1}+\beta_{i} \mathbf{p}_{i}$

In the current application, PCG will be terminated after a small number of iterations denoted $m-1$. At this point, we obtain an iterate $\mathbf{u}_{m}$ that did not necessarily converge to satisfy equilibrium, meaning

$$
\frac{\left\|\mathbf{f}-\mathbf{K} \mathbf{u}_{m}\right\|_{2}}{\|\mathbf{f}\|_{2}}=\frac{\left\|\mathbf{r}_{m}\right\|_{2}}{\|\mathbf{f}\|_{2}}>\epsilon
$$

Following the adjoint method, all the expressions involved in computing the PCG variables throughout the iterative procedure (until the termination step) should be multiplied by a corresponding adjoint variable and added to the objective function. Then the augmented objective functional for compliant mechanism design will have the form

$$
\begin{aligned}
\widehat{c}(\boldsymbol{\rho})= & \mathbf{l}^{T} \mathbf{u}_{m}+\widetilde{\mathbf{r}}_{1}^{T}\left(\mathbf{r}_{1}-\mathbf{f}+\mathbf{K} \mathbf{u}_{1}\right)+\widetilde{\mathbf{z}}_{1}^{T}\left(\mathbf{K}_{0} \mathbf{z}_{1}-\mathbf{r}_{1}\right)+ \\
& \widetilde{\mathbf{p}}_{1}^{T}\left(\mathbf{p}_{1}-\mathbf{z}_{1}\right)+\sum_{i=1}^{m-1} \widetilde{\alpha}_{i}\left(\alpha_{i}-\frac{\mathbf{r}_{i}^{T} \mathbf{z}_{i}}{\left(\mathbf{K} \mathbf{p}_{i}\right)^{T} \mathbf{p}_{i}}\right)+ \\
& \sum_{i=2}^{m} \widetilde{\mathbf{u}}_{i}^{T}\left(\mathbf{u}_{i}-\mathbf{u}_{i-1}-\alpha_{i-1} \mathbf{p}_{i-1}\right)+\sum_{i=2}^{m} \widetilde{\mathbf{r}}_{i}^{T}\left(\mathbf{r}_{i}-\mathbf{r}_{i-1}+\right. \\
& \left.\alpha_{i-1} \mathbf{K} \mathbf{p}_{i-1}\right)+\sum_{i=2}^{m-1} \widetilde{\mathbf{z}}_{i}^{T}\left(\mathbf{K}_{0} \mathbf{z}_{i}-\mathbf{r}_{i}\right)+ \\
& \sum_{i=1}^{m-2} \widetilde{\beta}_{i}\left(\beta_{i}-\frac{\mathbf{r}_{i+1}^{T} \mathbf{z}_{i+1}}{\mathbf{r}_{i}^{T} \mathbf{z}_{i}}\right)+ \\
& \sum_{i=2}^{m-1} \widetilde{\mathbf{p}}_{i}^{T}\left(\mathbf{p}_{i}-\mathbf{z}_{i}-\beta_{i-1} \mathbf{p}_{i-1}\right)
\end{aligned}
$$

When differentiating the augmented objective functional we wish to remain with explicit derivatives of the stiffness matrix and of the preconditioner only. For that purpose, we solve a set of adjoint equations that eliminates all other derivatives. The complete set of adjoint equations to be solved is presented in Amir et al. [2010], with only a minor difference due to the fact that it is derived there for a minimum compliance objective. We note that this approach leads to an iterative CG-like adjoint procedure that is performed in a reverse manner, beginning in the $m$-th 
cycle and ending in the first cycle. After performing the complete adjoint procedure, the design sensitivities include only explicit derivatives of the stiffness matrix and of the preconditioner that can be computed efficiently on an element level

$$
\begin{aligned}
\frac{\partial \widehat{c}}{\partial \rho_{e}}= & \widetilde{\mathbf{r}}_{1}^{T} \frac{\partial \mathbf{K}}{\partial \rho_{e}} \mathbf{u}_{1}+\sum_{i=1}^{m-1} \frac{\widetilde{\alpha}_{i} \mathbf{r}_{i}^{T} \mathbf{z}_{i}}{\left(\left(\mathbf{K}_{i}\right)^{T} \mathbf{p}_{i}\right)^{2}} \mathbf{p}_{i}^{T} \frac{\partial \mathbf{K}}{\partial \rho_{e}} \mathbf{p}_{i}+ \\
& \sum_{i=2}^{m} \alpha_{i-1} \widetilde{\mathbf{r}}_{i}^{T} \frac{\partial \mathbf{K}}{\partial \rho_{e}} \mathbf{p}_{i-1}+\sum_{i=1}^{m-1} \widetilde{\mathbf{z}}_{i}^{T} \frac{\partial \mathbf{K}_{0}}{\partial \rho_{e}} \mathbf{z}_{i}
\end{aligned}
$$

In summary, one approach is to keep the original formulation of the optimization problem that assumes an accurate solution of the nested analysis equations, and then use approximate design sensitivities. Another approach is to consider a slightly modified optimization problem that does not assume accurate solution of the nested equations and use consistent design sensitivities. In the following, the corresponding computational procedures are denoted 'variant 1' and 'variant 2' respectively. Clearly, implementing variant 2 makes more sense from a mathematical point of view but as shown in Section 4, this is not necessarily reflected in the numerical results.

\subsection{Choosing the reference design}

A successful application of approximate reanalysis in optimal design relies to a large extent on the choice of the reference stiffness matrix $\mathbf{K}_{0}$. In the case of the robust formulations, it is suggested to use the most dilated structure (within the considered range of dilation/erosion) as a reference structure. This holds also for the stochastic case where the uniformly dilated structure is not part of the set of designs to be considered in the optimization. Several arguments that support this particular choice are given in this section. The arguments are based on basic properties of the Combined Approximations approach as well as on observations arising in numerical experiments.

Convergence of the reanalysis series First we take a close look at the convergence of the series (11). Denoting $\mathbf{B} \equiv-\mathbf{K}_{0}^{-1} \Delta \mathbf{K}$, the series converges if and only if [Wilkinson, 1965; Kirsch, $2008]$

$$
\lim _{k \rightarrow \infty} \mathbf{B}^{k}=\mathbf{0}
$$

which in turn holds if and only if [Saad, 2003]

$$
\rho(\mathbf{B})<1 .
$$

The spectral radius is related to a consistent matrix norm of $\mathbf{B}$ by the inequality [Saad, 2003]

$$
\rho(\mathbf{B}) \leq\|\mathbf{B}\|
$$

meaning that a sufficient condition for convergence of the series is [Kirsch, 2008]

$$
\|\mathbf{B}\|<1 \text {. }
$$

Evaluating the errors involved in truncation of the series, the norm of the sum of additional terms beyond the first $s$ terms $\Delta \mathbf{u}=\mathbf{B}^{s}\left(\mathbf{I}+\mathbf{B}+\mathbf{B}^{2}+\mathbf{B}^{3}+\ldots\right) \mathbf{u}_{1}$ is bounded from above by

$$
\begin{gathered}
\|\Delta \mathbf{u}\|=\left\|\mathbf{B}^{s}\left(\sum_{k=0}^{\infty} \mathbf{B}^{k}\right) \mathbf{u}_{1}\right\| \leq\left\|\mathbf{B}^{s}\right\|\left\|\left(\sum_{k=0}^{\infty} \mathbf{B}^{k}\right) \mathbf{u}_{1}\right\| \leq \\
\left\|\mathbf{B}^{s}\right\|\left\|\sum_{k=0}^{\infty} \mathbf{B}^{k}\right\|\left\|\mathbf{u}_{1}\right\| \leq\|\mathbf{B}\|^{s} \frac{1}{1-\|\mathbf{B}\|}\left\|\mathbf{u}_{1}\right\|
\end{gathered}
$$

where we used properties of consistent matrix norms as well as the relations $\sum_{k=0}^{\infty} \mathbf{B}^{k}=(\mathbf{I}-\mathbf{B})^{-1}$ and $\left\|(\mathbf{I}-\mathbf{B})^{-1}\right\| \leq \frac{1}{1-\|\mathbf{B}\|}[$ Golub and Van Loan, 1996]. 
Relating the choice of $\mathbf{K}_{0}$ to convergence and errors In robust topology optimization a (potentially large) set of designs is evaluted simultaneously. Applying reanalysis techniques immediately raises the question of how to choose the reference design corresponding to $\mathbf{K}_{0}$ so that the most effective procedure is obtained. Throughout this section, we focus on a demonstrative scenario where two designs are to be evaluated. The main argument is that it is more effective to use the "stiffer" one as the reference design rather than vice-versa.

We first examine the impact on the condition (16) which can be elaborated as

$$
\|\mathbf{B}\|=\left\|-\mathbf{K}_{0}^{-1} \Delta \mathbf{K}\right\|<1 .
$$

We denote the stiffnesses of the two designs $\mathbf{K}_{1}$ and $\mathbf{K}_{2}$ and assume $\mathbf{K}_{1}$ is the "stiffer" design meaning $\mathbf{K}_{1} \succ \mathbf{K}_{2}$. $\mathbf{K}_{1}$ is chosen as the reference design so $\Delta \mathbf{K}=\mathbf{K}_{2}-\mathbf{K}_{1}$ and $\mathbf{K}_{1}-\mathbf{K}_{2}+\Delta \mathbf{K}=$ 0. Multiplying on both sides by $\mathbf{K}_{1}^{-1 / 2}$ gives

$$
\mathbf{I}-\mathbf{K}_{1}^{-1 / 2} \mathbf{K}_{2} \mathbf{K}_{1}^{-1 / 2}+\mathbf{K}_{1}^{-1 / 2} \Delta \mathbf{K K}_{1}^{-1 / 2}=\mathbf{0} .
$$

The matrix $\mathbf{K}_{1}^{-1 / 2} \mathbf{K}_{2} \mathbf{K}_{1}^{-1 / 2}$ is positive-definite because both $\mathbf{K}_{1}^{-1 / 2}$ and $\mathbf{K}_{2}$ are positive-definite. Therefore

$$
\mathbf{I}+\mathbf{K}_{1}^{-1 / 2} \Delta \mathbf{K K}_{1}^{-1 / 2} \succ \mathbf{0}
$$

or alternatively

$$
\mathbf{I} \succ-\mathbf{K}_{1}^{-1 / 2} \Delta \mathbf{K K}_{1}^{-1 / 2}
$$

On both sides of the inequality we have positive-definite matrices (because $-\Delta \mathbf{K}$ is positivedefinite) so it follows that for the matrix 2-norm (which is the spectral radius for Hermitian matrices [Saad, 2003]) we have

$$
\|\mathbf{I}\|_{2}>\left\|-\mathbf{K}_{1}^{-1 / 2} \Delta \mathbf{K K}_{1}^{-1 / 2}\right\|_{2}
$$

and using the relation $\left\|-\mathbf{K}_{1}^{-1 / 2} \Delta \mathbf{K K}_{1}^{-1 / 2}\right\|_{2}=\left\|-\mathbf{K}_{1}^{-1} \Delta \mathbf{K}\right\|_{2}$ (e.g. [Chen, 2005]) we obtain

$$
\left\|-\mathbf{K}_{1}^{-1} \Delta \mathbf{K}\right\|_{2}<1 \text {. }
$$

This means that convergence is guaranteed if $\mathbf{K}_{1}$ plays the role of the reference design, regardless of the magnitude of the differences in stiffness $\Delta \mathbf{K}$. An important consequence of (18) is that in design scenarios involving more than two structures, choosing the "stiffest" one as the reference design is a very effective choice because convergence is guaranteed for all evaluations of the other structures.

If $\mathbf{K}_{2}$ would have been chosen as the reference design, convergence could still be achieved but with dependence on the magnitude of $\Delta \mathbf{K}$. However, it is expected to be slower. The convergence rates for the choices of $\mathbf{K}_{1}$ or $\mathbf{K}_{2}$ as the reference design are related to $\left\|-\mathbf{K}_{1}^{-1} \Delta \mathbf{K}\right\|$ and $\left\|\mathbf{K}_{2}^{-1} \Delta \mathbf{K}\right\|$ respectively. The former is smaller because $\left\|\mathbf{K}_{1}^{-1}\right\|$ is smaller than $\left\|\mathbf{K}_{2}^{-1}\right\|$.

Let us now demonstrate the arguments given above on a single degree of freedom equilibrium equation $k u=f$, where $k$ is the stiffness, $u$ is the displacement and $f$ is the force. We consider the case of three available designs $k \in\{8,10,12\}$ and the aim is to compare the convergence of the reanalysis series for three scenarios, each involving a pair of designs. The approximate displacements computed using 10 series terms with various choices of $k$ and $k_{0}$ are plotted in Fig. 4(a). The relative absolute errors $\frac{\left|u_{e x a c t}-u_{s}\right|}{u_{e x a c t}}$ are plotted in Fig. 4(b). It can be seen that for stiffer reference designs $(\Delta k<0)$ the approximations approach the exact solution monotonously from below, while for softer reference designs $(\Delta k>0)$ fluctuations are observed. As expected, the convergence rates of the various series approximations are in complete correlation with the values of the ratio $\frac{|\Delta k|}{\left|k_{0}\right|}$ : the higher this ratio, the slower convergence is achieved. But the most important observation for the current application is the following: for evaluating any pair of 


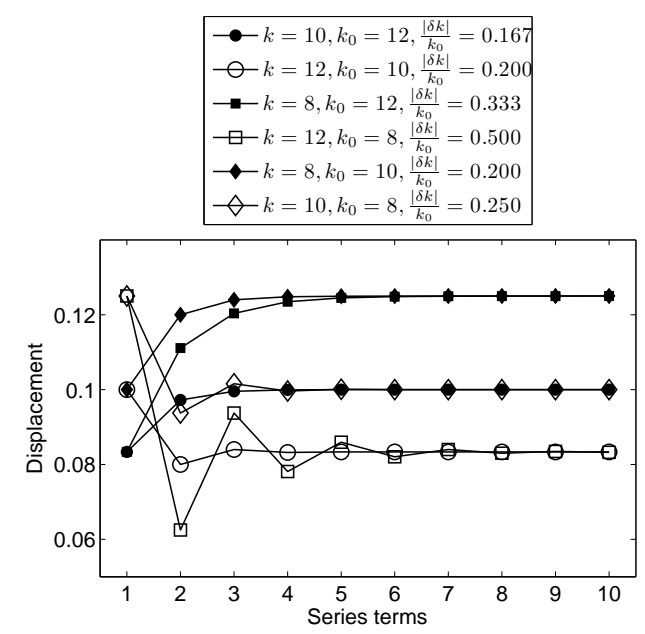

(a) Displacements computed using up to 10 series terms.

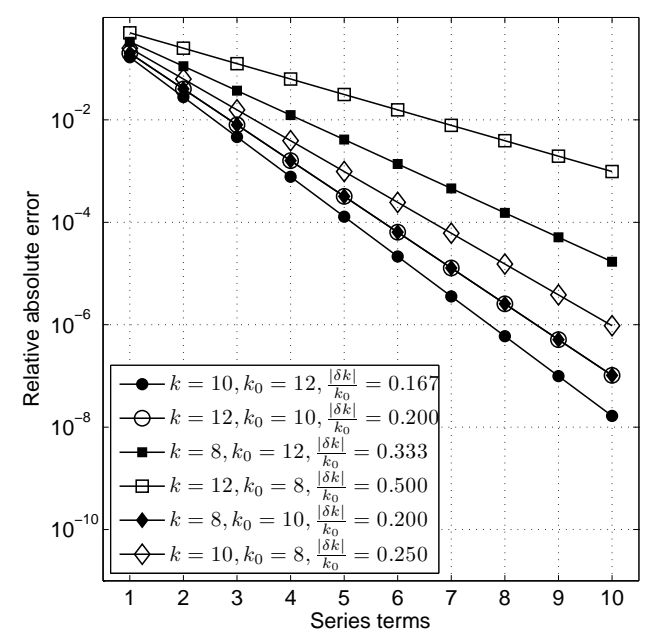

(b) Relative absolute errors of the approximation with up to 10 series terms. Convergence for $k=12$, $k_{0}=10$ coincides with $k=8, k_{0}=10$ because the ratios $\frac{|\Delta k|}{k_{0}}$ are the same.

Figure 4: Convergence of the reanalysis series in single degree of freedom problems. For any pair of designs, if the reference design and the re-analyzed design obey $k_{0}>k$ then convergence is faster compared to the case $k_{0}<k$.

designs, it is better to let the stiffer one play the role of $k_{0}$ because then the ratio $\frac{|\Delta k|}{\left|k_{0}\right|}$ is smaller, meaning convergence is faster. This will be further demonstrated in the following section, where a model problem of robust topology optimization is addressed.

Examining the bound (17), it can be seen that the truncation errors are reduced as $\|\mathbf{B}\|$ and $\left\|\mathbf{u}_{1}\right\|$ are reduced. Focusing again on the scenario involving two designs $\mathbf{K}_{1}$ and $\mathbf{K}_{2}$ with $\mathbf{K}_{1} \succ \mathbf{K}_{2},\|\mathbf{B}\|$ will be smaller if $\mathbf{K}_{1}$ plays the role of the reference design $\mathbf{K}_{0}$. Furthermore, the first series term $\left\|\mathbf{u}_{1}\right\|$ will also be smaller because the force vector is the same, giving $\left\|\mathbf{K}_{1}^{-1} \mathbf{f}\right\|<\left\|\mathbf{K}_{2}^{-1} \mathbf{f}\right\|$.

An important question arising is how to identify a "stiffer" design corresponding to a smaller $\left\|\mathbf{K}_{0}^{-1}\right\|$. In the context of the current application, all the designs are generated from the same "base" design (resulting from density filtering only) by either adding or removing linear elastic material. In the worst-case formulation, uniform dilation will result in a larger $\|\mathbf{K}\|$ and smaller $\left\|\mathbf{K}^{-1}\right\|$ compared to the base design as well as compared to the intermediate design. Erosion will result in a smaller $\|\mathbf{K}\|$ and larger $\left\|\mathbf{K}^{-1}\right\|$ compared to the base and intermediate designs. Therefore the dilated structure is the stiffest design available in the worst-case scenario.

In the stochastic formulation, material is added or removed according to the random distribution of the parameter $\eta$. Therefore it is difficult to classify which designs correspond to larger/smaller norms. However, it is clear that the uniformly dilated design has more added material than all the randomly generated designs - the dilation envelopes all other designs. Therefore it corresponds to a larger $\|\mathbf{K}\|$ and a smaller $\left\|\mathbf{K}^{-1}\right\|$ compared to the complete set of designs to be evaluated and can be identified as the stiffest design in the stochastic scenario as well. Choosing the stiffest design as $\mathbf{K}_{0}$ in the stochastic scenario is not necessarily the most efficient choice for some evaluations. Actually, the average magnitude of $\Delta \mathbf{K}$ is expected to be smaller if the intermediate (blueprint) design is used as reference. On the other hand, the value of $\|\mathbf{B}\|$ depends both on $\left\|\mathbf{K}_{0}^{-1}\right\|$ as well as on $\|\Delta \mathbf{K}\|$ so it is difficult to predict which reference design will eventually lead to the fastest convergence when the whole set of designs is considered. The limitation of using the intermediate design as reference is that convergence is 
not guaranteed when the evaluated design is stiffer, while using the stiffest design as reference ensures convergence for all designs. Furthermore, as will be shown in the following section, large errors are exhibited when evaluating a design that is stiffer than the reference. Therefore we conclude that using the stiffest design as reference gives a robust and effective procedure also in the framework of the stochastic approach.

Behavior of the PCG procedure The examination will now be extended to an actual case of robust topology optimization, where multiple structures are considered simultaneously. We focus on the convergence of the reanalysis procedure when it is formulated as a PCG, for different choices of the reference design. At this point it should be noted that the series terms from Eq. (11) are identical to the vectors in the Krylov subspace when the factors of $\mathbf{K}_{0}$ are employed as preconditioners [Kirsch et al., 2002]. In practice, convergence of the PCG (or CA) procedure is influenced by other parameters besides the quantity $\|\mathbf{B}\|$; in some cases convergence is achieved even if $\|\mathbf{B}\|$ is large [Kirsch, 2008]. This is because the approximation is not computed directly from the series (11) but rather from a reduced basis solution. Nevertheless, it will be shown that the behavior of the PCG/CA is procedure is essentially the same as that of the single degree of freedom system, where reanalysis was based solely on the series terms.

The test case considers uniform manufacturing errors represented by dilation and erosion operators and the problem formulation is given in (3). Five designs are considered, corresponding to different deterministic values of the parameter $\eta$ that governs the dilation/erosion via the projection functions (1) or (2). For the purpose of a qualitative investigation, a "snapshot" of the design process is taken after 200 cycles, see Figure 5 . The linear systems are numbered from 1 to 5 , the former corresponding to the most dilated structure $(\eta=0.3$, Figure $5(\mathrm{~b}))$ and the latter corresponding to the most eroded structure $(\eta=0.7$, Figure $5(\mathrm{f}))$. We attempt to solve all five linear systems with all available factorizations as preconditioners, meaning four PCG procedures for each linear system. The convergence tolerance in terms of the relative residual norm is set to $1 e-10$ and the maximum number of PCG iterations is set to 1000. The number of iterations required for convergence is given in Table 1 where 1000 means that PCG did not converge to the required tolerance. The convergence rate of PCG is often related to the condition number of the preconditioned system, so these are given in Table 2 .

Table 1: PCG iterations

\begin{tabular}{l|ccccc} 
& \multicolumn{5}{|c}{ Reanalysed design } \\
\hline Reference design & $\# 1, \eta=0.3$ & $\# 2, \eta=0.4$ & $\# 3, \eta=0.5$ & $\# 4, \eta=0.6$ & $\# 5, \eta=0.7$ \\
\hline$\# 1, \eta=0.3$ & \multicolumn{7}{c}{66} & 435 & 1000 & 1000 \\
\hline$\# 2, \eta=0.4$ & 95 & & 64 & 435 & 1000 \\
\hline$\# 3, \eta=0.5$ & 783 & 95 & & 63 & 432 \\
\hline$\# 4, \eta=0.6$ & 1000 & 779 & 91 & & 62 \\
\hline$\# 5, \eta=0.7$ & 1000 & 1000 & 718 & 93
\end{tabular}

Table 2: Condition numbers

\begin{tabular}{|c|c|c|c|c|c|}
\hline & \multicolumn{5}{|c|}{ Reanalysed design } \\
\hline Reference design & $\# 1, \eta=0.3$ & $\# 2, \eta=0.4$ & $\# 3, \eta=0.5$ & $\# 4, \eta=0.6$ & $\# 5, \eta=0.7$ \\
\hline$\# 1, \eta=0.3$ & & $4.39 \mathrm{e} 2$ & $5.69 \mathrm{e} 4$ & $5.79 \mathrm{e} 6$ & $1.84 \mathrm{e} 8$ \\
\hline$\# 2, \eta=0.4$ & $7.87 \mathrm{e} 2$ & & $4.17 \mathrm{e} 2$ & $5.22 \mathrm{e} 4$ & $4.87 \mathrm{e} 6$ \\
\hline$\# 3, \eta=0.5$ & $1.24 \mathrm{e} 5$ & $8.11 \mathrm{e} 2$ & & $4.01 \mathrm{e} 2$ & $5.60 \mathrm{e} 4$ \\
\hline$\# 4, \eta=0.6$ & $1.00 \mathrm{e} 7$ & $8.61 \mathrm{e} 4$ & $7.11 \mathrm{e} 2$ & & $4.49 \mathrm{e} 2$ \\
\hline$\# 5, \eta=0.7$ & $2.49 \mathrm{e} 8$ & $8.53 \mathrm{e} 6$ & $1.06 \mathrm{e} 5$ & $7.60 \mathrm{e} 2$ & \\
\hline
\end{tabular}

Several immediate observations arise when examining Tables 1 and 2. First, for differences in $\eta$ larger than 0.1, the condition numbers are relatively high and the PCG procedures appear 


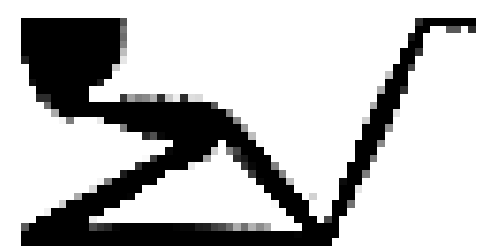

(a)

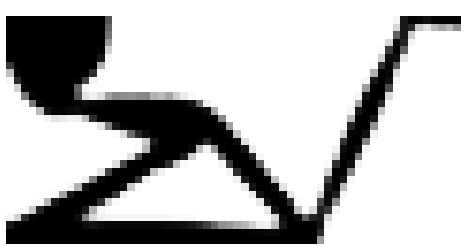

(d)

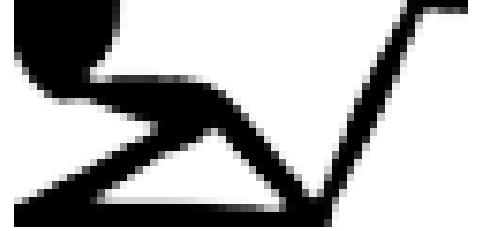

(b)

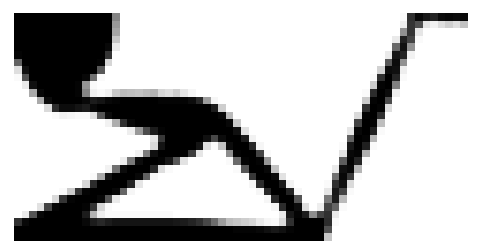

(e)

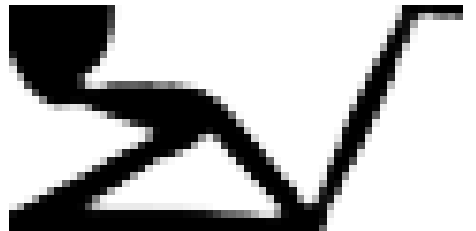

(c)

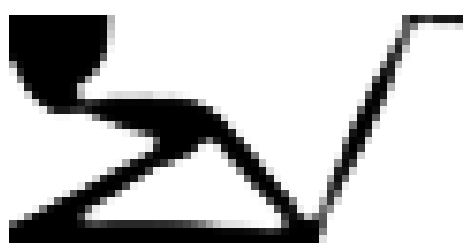

(f)

Figure 5: Example involving the robust design of a force inverter, demonstrating the choice of the reference design. Uniform errors are considered in the from of dilation / erosion operations. The figures represent the following data sets after 200 design cycles: (a) Design variables; (b) Most dilated structure, $\eta=0.3$; (c) Mildly dilated structure, $\eta=0.4$; (d) Intermediate structure, $\eta=0.5$; (d) Mildly eroded structure, $\eta=0.6$; (f) Most eroded structure, $\eta=0.7$.

to converge very slowly. Second, for any given difference in $\eta$, it seems to be beneficial to use the stiffer structure as the preconditioner: in both tables the upper triangle terms represent a better performance than the lower triangle terms. However, the differences are not dramatic and by no means support the choice of the stiffest structure (system 1) as the reference design. In fact, if a decision were to be taken according to the information in Tables 1 and 2, the most sensible choice would be to use the intermediate structure (system 3) as the reference design.

A closer look at the short-term convergence of the PCG solutions leads to a different conclusion which is perfectly compatible with the conclusions arising from the examination of the single degree-of-freedom system. The following examination is limited to the first 50 PCG iterations performed when solving systems 1,3 and 5 with the preconditioners chosen from the same systems. The exclusion of systems 2 and 4 from the discussion is for demonstrative purposes only and it is noted that the same behavior was observed also when considering the full set of systems. We focus on the short-term behavior because the aim is to perform very few PCG iterations for each reanalysis problem; otherwise a factorization is more efficient. The convergence is measured using two different quantities: 1) The relative norm of residual forces; 2) The error (compared to the exact solution) in the value of the quadratic functional $\phi=\frac{1}{2} \mathbf{u}^{T} \mathbf{K u}-\mathbf{f}^{T} \mathbf{u}$, which is the quantity that is minimized by PCG.

Examining the results plotted in Figure 6, it is clearly beneficial to use a stiffer structure as the reference design: the short-term reduction in errors is significantly better than when using a preconditioner corresponding to a design that is more flexible than the design to be evaluated. Moreover, the short-term performance is better with a stiff preconditioner even if the difference in stiffness $\Delta \mathbf{K}$ is large. For example, when solving system 5 with the factors of system 1 as preconditioners, the errors decrease rapidly; whereas when solving system 3 with the factors of system 5 as preconditioners, the errors decrease slowly over the first 50 iterations. This is despite the better condition number of the latter procedure.

In conclusion, we argue that the most dilated structure should be used as the reference design for reanalysis within both robust formulations discussed in this article. Furthermore, the numerical evidence presented in this section also explains the relatively good performance of the 


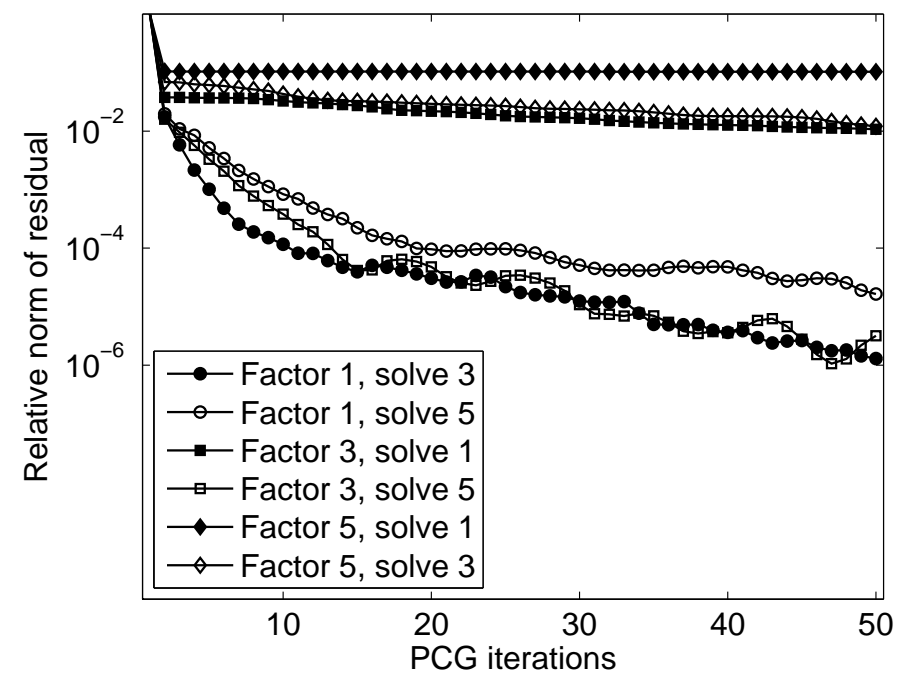

(a) Relative norms of residuals.

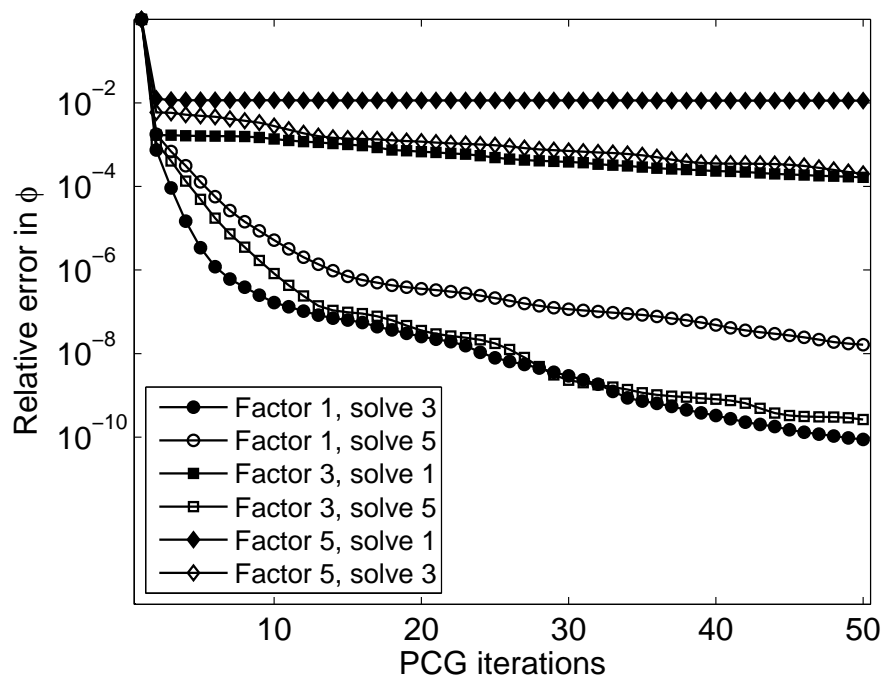

(b) Relative error in the PCG functional $\phi$.

Figure 6: Convergence of the reanalysis / PCG procedure with different factorizations as preconditioners. 
procedure described in Amir and Sigmund [2011], where a solid domain is used as the reference design for the whole design process in standard topology optimization.

\subsection{Implementation in robust formulations}

The computational procedures for robust topology optimization based on approximate reanalysis are hereby outlined. We address two approaches to robust design: the worst case formulation and the stochastic formulation. The formulation of the optimization problems is the same as presented in (3) and (8), except for the introduction of approximations in the solution of the nested analysis equations. In the worst case approach we consider

$$
\begin{aligned}
\mathbf{K}\left(\overline{\widetilde{\boldsymbol{\rho}}}^{d}\right) \mathbf{u}^{d} & =\mathbf{f} \\
\mathbf{K}\left(\overline{\widetilde{\boldsymbol{\rho}}}^{i}\right) \mathbf{u}^{i} & \approx \mathbf{f} \\
\mathbf{K}\left(\overline{\widetilde{\boldsymbol{\rho}}}^{e}\right) \mathbf{u}^{e} & \approx \mathbf{f}
\end{aligned}
$$

meaning that the analysis of the dilated structure is accurate while the analyses of the intermediate and eroded structures are approximate. In the stochastic approach, a uniformly dilated structure does not belong to the set of designs to be evaluated; it is generated only to serve as a "reference design" for reanalysis. Therefore the nested problem has the form

$$
\mathbf{K}(\boldsymbol{\rho}, \boldsymbol{\xi}) \mathbf{u}(\boldsymbol{\rho}, \boldsymbol{\xi}) \approx \mathbf{f}
$$

meaning that approximate reanalysis is performed for all the randomly generated designs. Into each of these approaches we integrate two variants of approximate reanalysis. In the first variant, reanalysis is performed on both the analysis problem and the adjoint problem, resulting in an approximate sensitivity analysis, see Eq. (14). In the second variant, reanalysis is performed on the analysis problem only and then consistent design sensitivities are derived, see Eq. (15).

Worst case approach Demonstrated on the case of three designs (dilated, intermediate and eroded), the procedure to be repeated within each design cycle until convergence is as follows:

1. Compute factors of $\mathbf{K}_{0}$ corresponding to the dilated structure

(a) $\mathbf{K}_{0}=\mathbf{K}\left(\overline{\widetilde{\boldsymbol{\rho}}}^{d}\right)$

(b) $\mathbf{U}_{0}=\operatorname{chol}\left(\mathbf{K}_{0}\right)$

2. Solve $\mathbf{K}\left(\overline{\widetilde{\boldsymbol{\rho}}}^{d}\right)\left[\mathbf{u}^{d} \boldsymbol{\lambda}^{d}\right]=[\mathbf{f} \mathbf{l}]$ for the dilated design.

3. Variant 1: Compute the approximations $\left[\widetilde{\mathbf{u}}^{i} \widetilde{\boldsymbol{\lambda}}^{i}\right]$ and $\left[\widetilde{\mathbf{u}}^{e} \widetilde{\boldsymbol{\lambda}}^{e}\right]$ by performing a few blockPCG iterations on $\mathbf{K}\left(\overline{\widetilde{\boldsymbol{\rho}}}^{i}\right)\left[\mathbf{u}^{i} \boldsymbol{\lambda}^{i}\right]=[\mathbf{f} \mathbf{l}]$ and $\mathbf{K}\left(\overline{\widetilde{\boldsymbol{\rho}}}^{e}\right)\left[\mathbf{u}^{e} \boldsymbol{\lambda}^{e}\right]=[\mathbf{f} \mathbf{l}]$; use the factors of $\mathbf{K}_{0}$ as preconditioners; use $\left[\widetilde{\mathbf{u}}^{i} \widetilde{\boldsymbol{\lambda}}^{i}\right]$ and $\left[\widetilde{\mathbf{u}}^{e} \widetilde{\boldsymbol{\lambda}}^{e}\right]$ from the previous design cycle as initial guesses. Variant 2: Compute the approximations $\widetilde{\mathbf{u}}^{i}$ and $\widetilde{\mathbf{u}}^{e}$ by performing a few PCG iterations on $\mathbf{K}\left(\overline{\widetilde{\boldsymbol{\rho}}}^{i}\right) \mathbf{u}^{i}=\mathbf{f}$ and $\mathbf{K}\left(\overline{\widetilde{\boldsymbol{\rho}}}^{e}\right) \mathbf{u}^{e}=\mathbf{f}$; use the factors of $\mathbf{K}_{0}$ as preconditioners; use $\widetilde{\mathbf{u}}^{i}$ and $\widetilde{\mathbf{u}}^{e}$ from the previous design cycle as initial guesses.

4. Compute design sensitivities for the dilated structure using Eq. (5) followed by the chain rule (6).

5. Variant 1: Compute approximate design sensitivities for the intermediate and eroded structures, as in Eq. (14) followed by the chain rule (6).

Variant 2: Compute consistent design sensitivities for the intermediate and eroded structures following the procedure discussed in Section 3.2. When applying the chain rule to 
obtain the sensitivities with respect to the design variables, note that the preconditioner depends on the dilated projection while the stiffness matrix depends on the intermediate or eroded projection.

6. Update the design variables $\boldsymbol{\rho}$ by solving a min-max problem using MMA.

Stochastic approach Similarly, in the stochastic approach the procedure to be repeated within each design cycle until convergence is as follows:

1. Compute factors of $\mathbf{K}_{0}$ corresponding to a uniformly dilated structure with $\eta=\eta_{\min }$

(a) $\mathbf{K}_{0}=\mathbf{K}\left(\overline{\widetilde{\boldsymbol{\rho}}}^{d}\right)$

(b) $\mathbf{U}_{0}=\operatorname{chol}\left(\mathbf{K}_{0}\right)$

2. Variant 1: For each of the events represented by the random vector $\boldsymbol{\xi}(\theta)$, compute the approximation $[\widetilde{\mathbf{u}} \widetilde{\boldsymbol{\lambda}}]$ by performing a few block-PCG iterations on $\mathbf{K}(\boldsymbol{\rho}, \boldsymbol{\xi})[\mathbf{u} \boldsymbol{\lambda}]=[\mathbf{f} \mathbf{l}]$; use the factors of $\mathbf{K}_{0}$ as preconditioners; use $[\widetilde{\mathbf{u}} \tilde{\boldsymbol{\lambda}}]$ corresponding to the same event in the previous design cycle as an initial guess.

Variant 2: For each of the events represented by the random vector $\boldsymbol{\xi}(\theta)$, compute the approximation $\widetilde{\mathbf{u}}$ by performing a few PCG iterations on $\mathbf{K}(\boldsymbol{\rho}, \boldsymbol{\xi}) \mathbf{u}=\mathbf{f}$; use the factors of $\mathbf{K}_{0}$ as preconditioners; use $\widetilde{\mathbf{u}}$ corresponding to the same event in the previous design cycle as an initial guess.

3. Variant 1: For each of the events represented by the random vector $\boldsymbol{\xi}(\theta)$, compute approximate design sensitivities as in Eq. (14) followed by the chain rule (6).

Variant 2: For each of the events represented by the random vector $\boldsymbol{\xi}(\theta)$, compute consistent design sensitivities following the procedure discussed in Section 3.2. When applying the chain rule to obtain the sensitivities with respect to the design variables, note that the preconditioner depends on the most dilated projection while the stiffness matrix depends on the particular random projection.

4. Compute the sensitivities of the stochastic objective and volume constraint as described in Section 2.2.

5. Update the design variables $\boldsymbol{\rho}$ using MMA.

\section{Examples}

In this section we present several results of robust topology optimization based on efficient reanalysis. The main purpose is to examine whether manufacturing-tolerant designs can be obtained while avoiding the exact solution of the finite element analyses, thus achieving a satisfactory qualitative result in an efficient manner. Furthermore, we would like to gain insight regarding the trade-off between the accuracy and the efficiency of our proposed approach. For a fair comparison, the settings of the example problems follow directly those presented by Wang et al. [2011] and Schevenels et al. [2011].

Two robust design problems are considered: a force inverter design using the worst case approach and a gripper design using the stochastic approach. The problems are solved with various resolutions of the reanalysis procedure, represented by the maximum allowed number of PCG iterations which varies between 1 to 10. Both implementations of reanalysis techniques denoted 'variant 1' and 'variant 2' are examined. Each optimization problem is solved with a fixed number of 300 design cycles. Within each cycle, an approximate sub-problem is generated and solved by the Method of Moving Asymptotes - MMA [Svanberg, 1987]. In all examples, the initial design is a uniform distribution of material that satisfies the volume constraint. 
The projection functions are driven gradually towards a true Heaviside step function by doubling the value of the parameter $\beta$ every 50 design cycles, beginning with 1 and ending with 32 . In the context of applying the reanalysis approach, every 'jump' in $\beta$ poses an obstacle because of the significant changes to the projected density distribution at these cycles. Consequently, the initial guess for the PCG procedure may be a bad starting point and the accuracy of reanalysis could be unsatisfactory. In order to overcome this difficulty, a maximum number of 10 PCG iterations is allowed every time $\beta$ is increased, regardless of the number of PCG iterations allowed in the particular experiment. The additional computational cost is relatively low because this safety measure is taken only once every 50 design cycles.

We note that there is a strong relation between the continuation scheme governing the value of the parameter $\beta$ and the expected accuracy of reanalysis. For low values of $\beta$, the projection functions for various values of the threshold $\eta$ are similar. This means that in the first stages of the optimization, we are basically evaluating a set of very similar designs. Consequently, the errors associated with approximate reanalysis are relatively small in the first 50 to 100 design cycles. In fact, it is observed that for the first 50 design cycles when $\beta=1$, reanalysis with 5-6 PCG iterations yields an accurate solution. As $\beta$ is increased, also the errors due to the approximate evaluation of the response become larger.

\subsection{Example 1: force inverter design, worst case formulation}

In this example we aim at reproducing one of the results reported by Wang et al. [2011] using the reanalysis-based procedure. In particular, we refer to the robust design of a force inverter (see Section 2.1) where the dilated, intermediate and eroded designs correspond to the threshold values $\eta=0.3, \eta=0.5$ and $\eta=0.7$ respectively. The problem is solved on a $200 \times 100 \mathrm{FE}$ mesh (square 4-node, bi-linear elements) and the density filter radius is 5.6 times the length of a single element. For further details regarding the problem setting the reader is referred to Wang et al. [2011].

In the design of a force inverter, it is well-known that a strong local minimum is encountered in the first design cycles when the output displacement changes sign from positive to negative. In order to avoid this local minimum, the min-max formulation is replaced by a simple sum of the three objectives for the first 10 design cycles in both implementations by Sigmund [2009] and Wang et al. [2011]. Accordingly, when using reanalysis procedures, a maximum number of 10 PCG iterations is allowed for the first 10 design cycles. This means that an accurate analysis is achieved in practice because at these early design cycles only up to 6 PCG iterations were required to reach the tolerance of $1 e-6$ in terms of relative residual forces.

Optimized designs obtained using efficient reanalysis are shown and compared to those generated with full factorizations in Figures 7 and 8. Examining the various layouts, it can be clearly seen that even rough approximations (corresponding to very few PCG reanalysis iterations) are sufficiently accurate in order to satisfy the objectives of robust topology optimization: 1) The optimized designs are robust with respect to over- and under-etching; 2) Local length scale control is achieved on both solid and void regions of the intermediate design; and 3) Crisp black and white designs are obtained.

The performance of the optimized inverters in terms of output displacement is presented in Table 3. Opposite to standard expectations, variant 1 involving approximate sensitivity analysis yields results closer to those obtained with the full solves, compared to variant 2 where consistent sensitivities are derived. This can be seen by examining both the output displacements as well as the norms of the differences in design variables, shown in Table 4. With approximate sensitivity analysis, all the results are practically identical to the results using three full solves. This demonstrates the tolerance of the optimization program towards inexact gradient information, a phenomenon that was observed also in previous studies on efficient topology optimization procedures [Amir et al., 2010; Amir and Sigmund, 2011]. With consistent sensitivity analysis, the reanalysis approach appears to converge towards the result of the full procedure as the number 

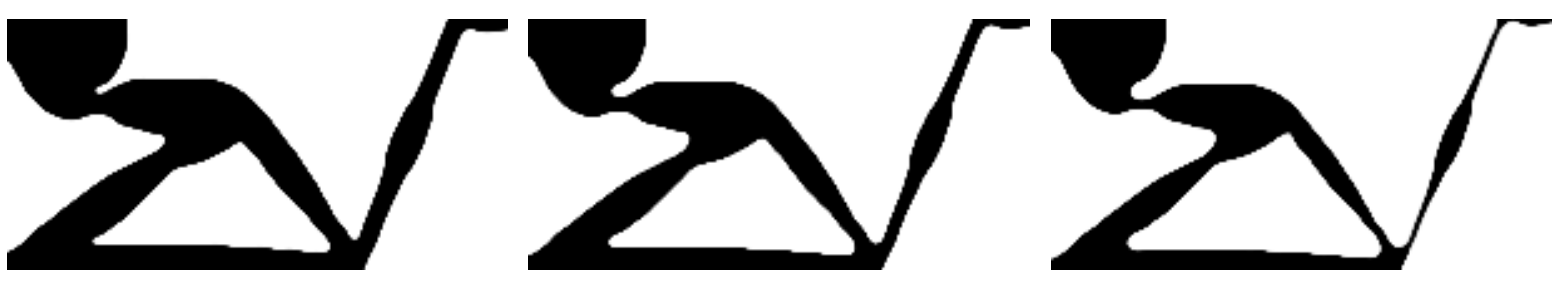

(a) Optimized designs obtained with a reanalysis procedure, 1 PCG iteration. $f=\{-2.144,-2.307,-2.144\}$
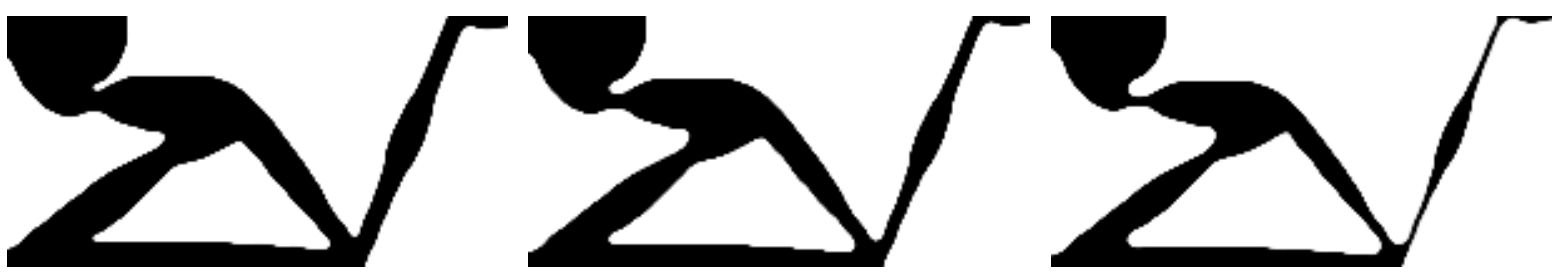

(b) Optimized designs obtained with a reanalysis procedure, 3 PCG iterations. $f=\{-2.143,-2.306,-2.143\}$
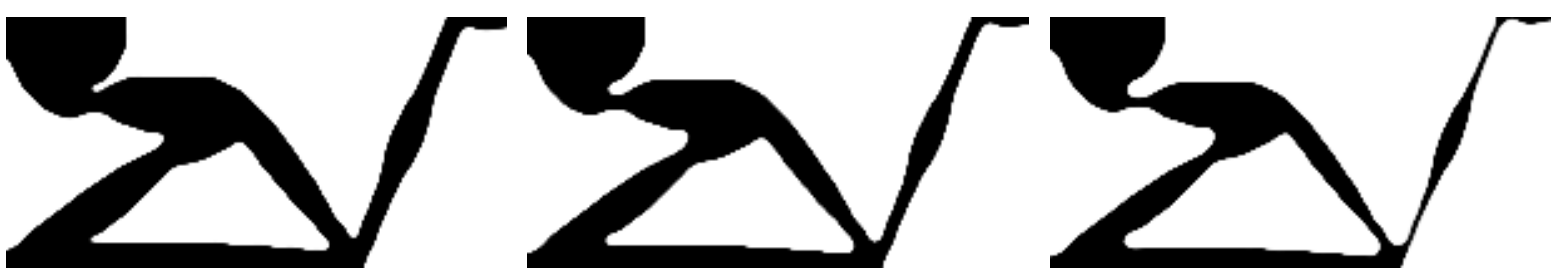

(c) Optimized designs obtained with a reanalysis procedure, 5 PCG iterations. $f=\{-2.143,-2.307,-2.143\}$
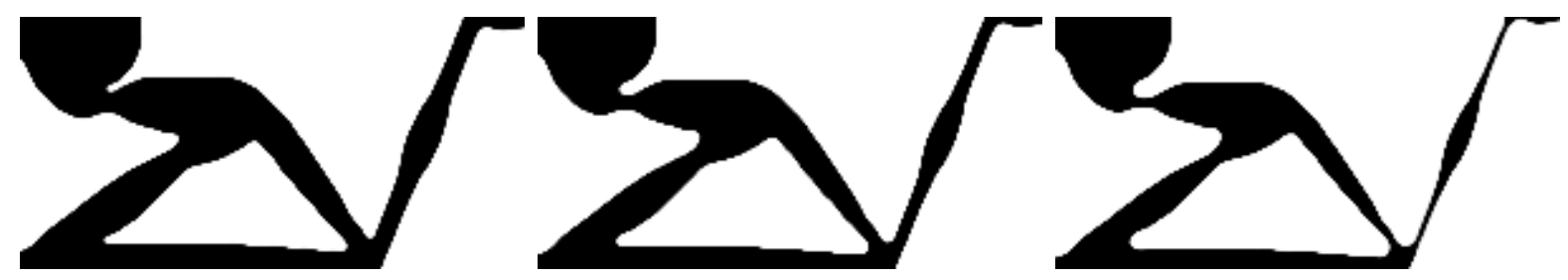

(d) Optimized designs obtained with a standard procedure. $f=\{-2.144,-2.308,-2.144\}$

Figure 7: Optimized layouts and performance of robust force inverters obtained using reanalysis procedures with approximate sensitivity analysis. In the bottom figure, results of the standard procedure are presented for comparison. 


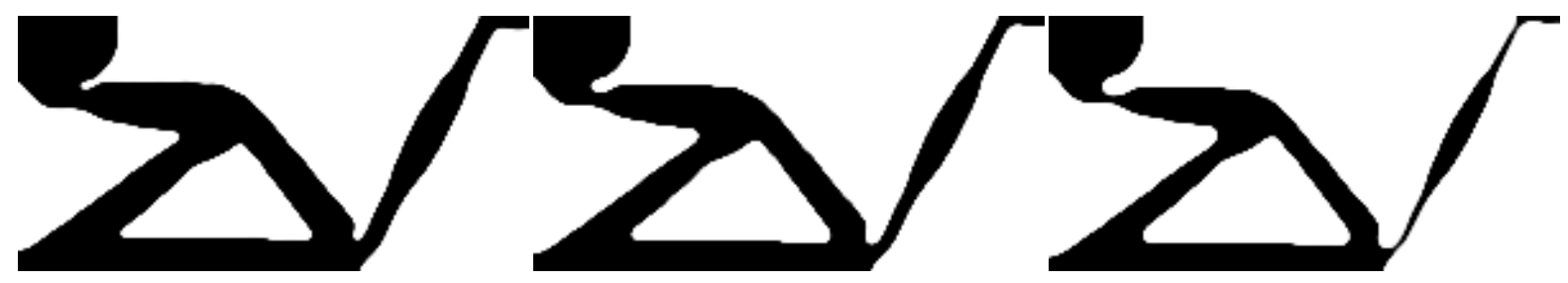

(a) Optimized designs obtained with a reanalysis procedure, 1 PCG iteration. $f=\{-1.960,-2.104,-1.961\}$

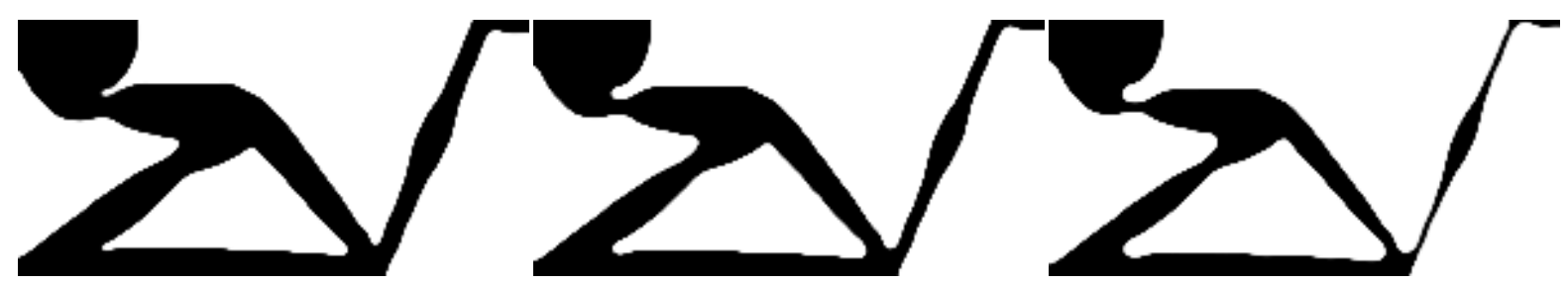

(b) Optimized designs obtained with a reanalysis procedure, 3 PCG iterations. $f=\{-2.132,-2.294,-2.132\}$

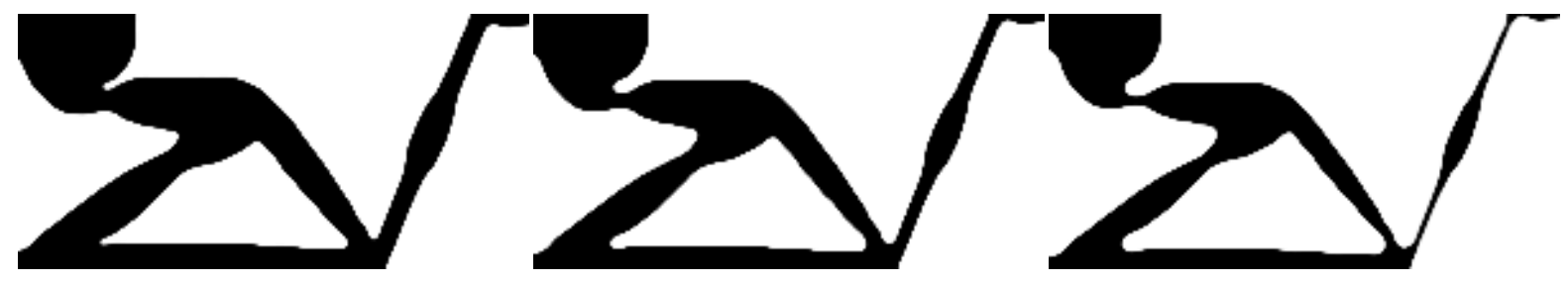

(c) Optimized designs obtained with a reanalysis procedure, 5 PCG iterations. $f=\{-2.135,-2.303,-2.135\}$

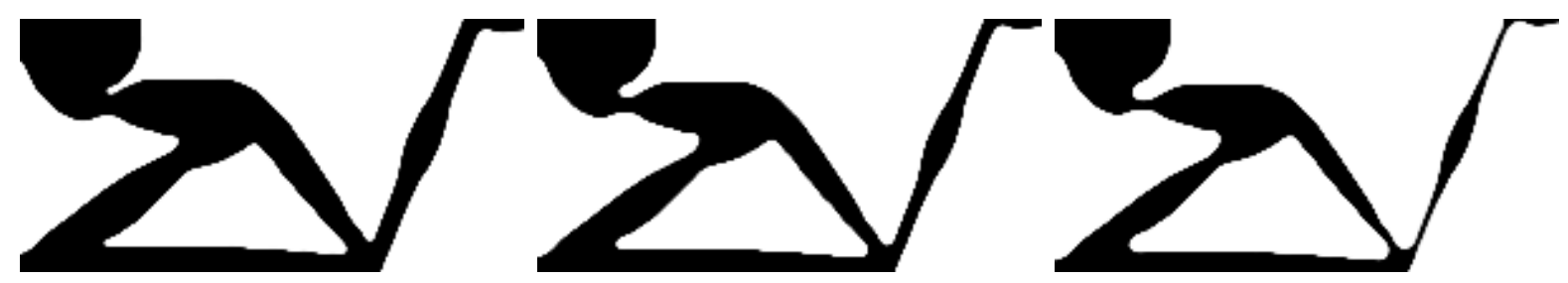

(d) Optimized designs obtained with a standard procedure. $f=\{-2.144,-2.308,-2.144\}$

Figure 8: Optimized layouts and performance of robust force inverters obtained using reanalysis procedures with consistent sensitivity analysis. In the bottom figure, results of the standard procedure are presented for comparison. 
of PCG iterations is increased. When only 1 PCG iteration is performed, a large difference in the value of the final objective is observed $-8.6 \%$ compared to the full procedure. This can also be seen in the optimized layouts which have the same topology but differ in shape. Nevertheless, when 2 or more PCG iterations are used, the differences in objective function become rather small and the final layouts and objectives are almost identical to those corresponding to the full procedure.

Table 3: Output displacement of the force inverter

\begin{tabular}{l|ccc|ccc} 
& \multicolumn{3}{|c|}{ Variant 1 } & \multicolumn{3}{c}{ Variant 2} \\
\hline PCG iterations & $\eta=0.3$ & $\eta=0.5$ & $\eta=0.7$ & $\eta=0.3$ & $\eta=0.5$ & $\eta=0.7$ \\
\hline 1 & -2.144 & -2.307 & -2.144 & -1.960 & -2.104 & -1.961 \\
2 & -2.141 & -2.307 & -2.141 & -2.112 & -2.275 & -2.112 \\
3 & -2.143 & -2.306 & -2.143 & -2.132 & -2.294 & -2.132 \\
4 & -2.141 & -2.307 & -2.139 & -2.129 & -2.298 & -2.129 \\
5 & -2.143 & -2.307 & -2.143 & -2.135 & -2.303 & -2.135 \\
10 & -2.146 & -2.311 & -2.146 & -2.143 & -2.308 & -2.143 \\
\hline Direct solves & -2.144 & -2.308 & -2.144 & -2.144 & -2.308 & -2.144
\end{tabular}

Table 4: Differences in optimized designs of the force inverter

\begin{tabular}{l|c|c} 
PCG iterations & $\left\|\boldsymbol{\rho}-\boldsymbol{\rho}_{\text {std }}\right\|$, variant 1 & $\left\|\boldsymbol{\rho}-\boldsymbol{\rho}_{\text {std }}\right\|$, variant 2 \\
\hline 1 & 12.1606 & 47.2594 \\
2 & 15.4202 & 33.8070 \\
3 & 10.7955 & 24.8957 \\
4 & 11.0450 & 23.0477 \\
5 & 10.1269 & 20.4201 \\
10 & 9.8739 & 17.2166
\end{tabular}

As pointed out above, the optimization program seems to be rather insensitive to inaccuracies in the gradients supplied to it. Accordingly, the implementation of variant 1 leads to better results than that of variant 2 . The approximation errors associated with employing reanalysis are plotted in Figure 9. As observed also in previous studies, the errors in primal (analysis) equations are significantly smaller than in the adjoint equations, presumably due to the problem setting. Nevertheless, the outcome of the optimization process is accurate despite errors in the order of up to $10 \%$ (in terms of the relative norm of the residual) in the solution of the adjoint problem.

Robustness of the design is achieved with all approximate solutions. This can be observed by examining Figure 10, where the actual performance (output displacement) of the designs for a wide range of erosion / dilation operations is depicted. The designs utilized for this purpose are those generated using consistent sensitivity analysis. Nevertheless, the same behavior is expected when evaluating the designs obtained with approximate sensitivities. In fact, judging by the results in Table 3 and by the layouts in Figure 7, they should perform even better.

\subsection{Example 2: compliant gripper design, stochastic formulation}

In this example we aim at reproducing one of the results reported by Schevenels et al. [2011] using reanalysis-based procedures. In particular, we refer to the robust design of a gripper mechanism (see Section 2.2), taking into account non-uniform spatially varying errors. Over- and underetching are represented by randomly varying the threshold value $\eta$ in the range $0.4 \leq \eta \leq 0.6$. For the optimization process, the mean and standard deviation of the gripping performance are estimated by means of a Monte Carlo simulation with 100 random realizations. Using the 


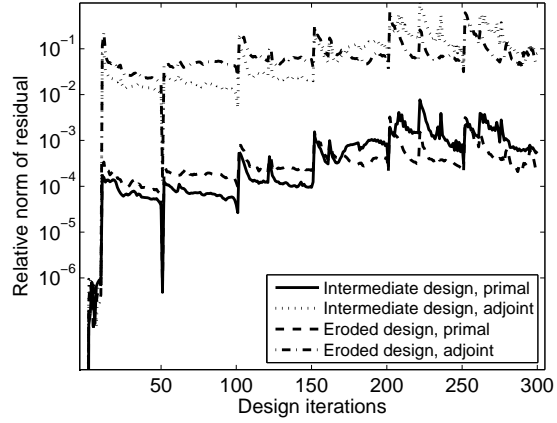

(a) Reanalysis with 1 block-PCG iteration.

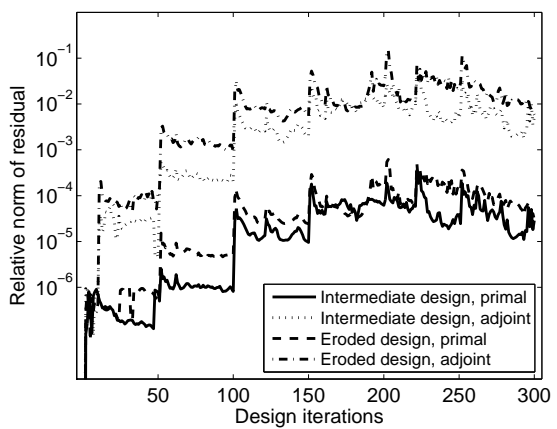

(c) Reanalysis with 5 block-PCG iterations.

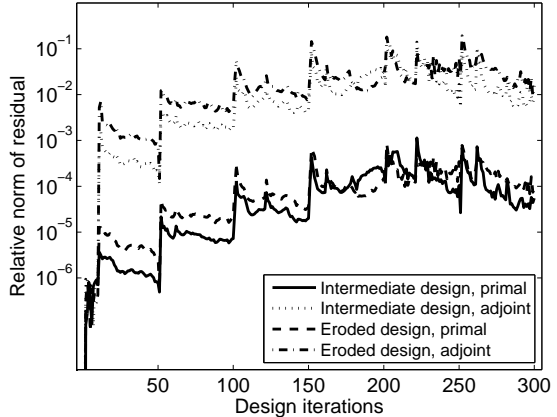

(b) Reanalysis with 3 block-PCG iterations.

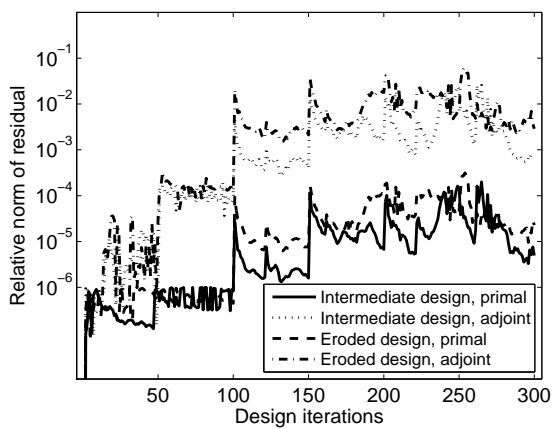

(d) Reanalysis with 10 block-PCG iterations.

Figure 9: Approximation errors associated with the reanalysis procedure throughout 300 design cycles of the force inverter. Errors are measured in terms of the relative norms of residual forces.

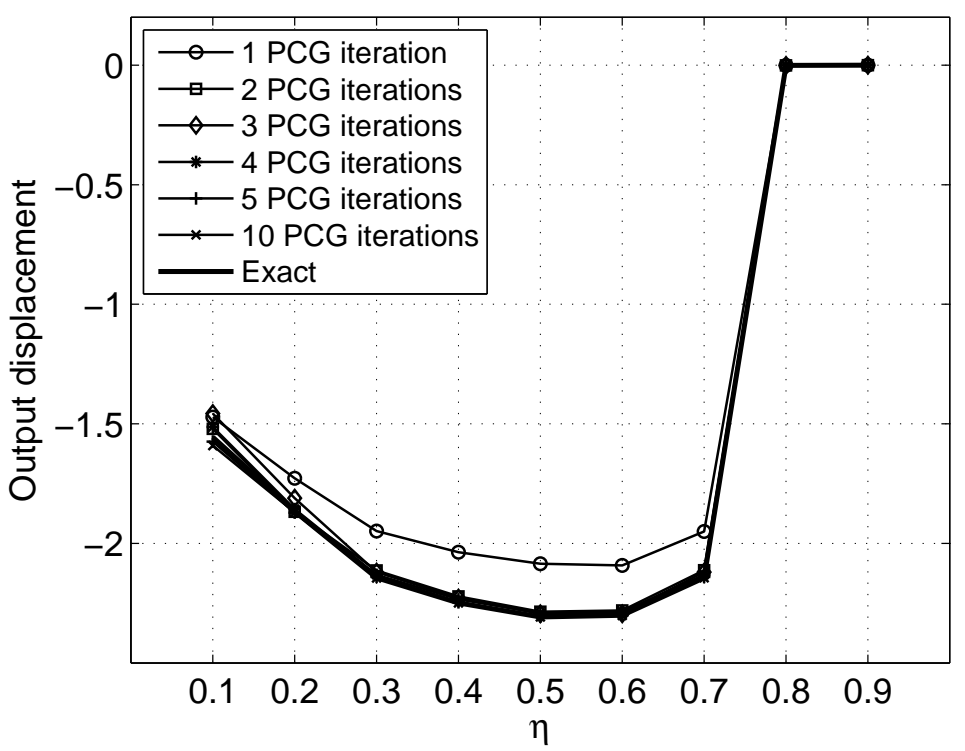

Figure 10: Actual performance of the optimized force inverters using consistent sensitivity analysis. Optimization considered only $\eta=$ $\{0.3,0.5,0.7\}$ but robust performance is achieved for a wider range of uniform errors simulated by $0.1 \leq \eta \leq 0.7$, even with rough approximations in the reanalysis. All optimized designs fail when excessive erosion $(\eta>0.7)$ is considered. 
optimized 'blueprint' design, a finer Monte Carlo simulation is performed with 3000 random samples to obtain a more accurate estimate of the mean and standard deviation. The problem is solved on a $200 \times 200 \mathrm{FE}$ mesh and the density filter radius is 8.4 times the length of a single element. For further details regarding the problem setting the reader is referred to Schevenels et al. [2011].

Optimized designs obtained using efficient reanalysis are shown and compared to those generated by standard procedures in Figures 11 and 12. The layouts represent the blueprint design corresponding to a uniform projection with a threshold $\eta=0.5$. As was the case for the robust force inverter, also this example demonstrates that approximate procedures are capable of generating robust designs with local length scale control. The layouts corresponding to a reanalysis-based optimization are practically identical to that obtained using the full procedure. Even with only a single PCG iteration, a hinge-free robust design is generated, though its performance is not as good as of the other designs.

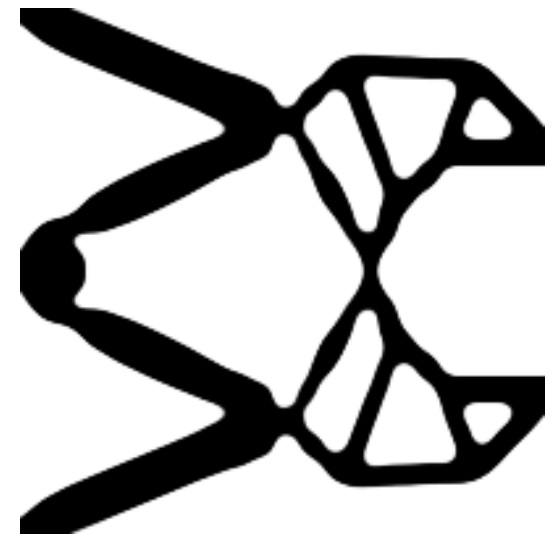

(a) Optimized designs obtained with a reanalysis procedure, 1 PCG iteration. $f_{0}=-1.8582, m_{f}=-1.7859, \sigma_{f}=$ $0.068, C I=(-1.7883,-1.7835)$

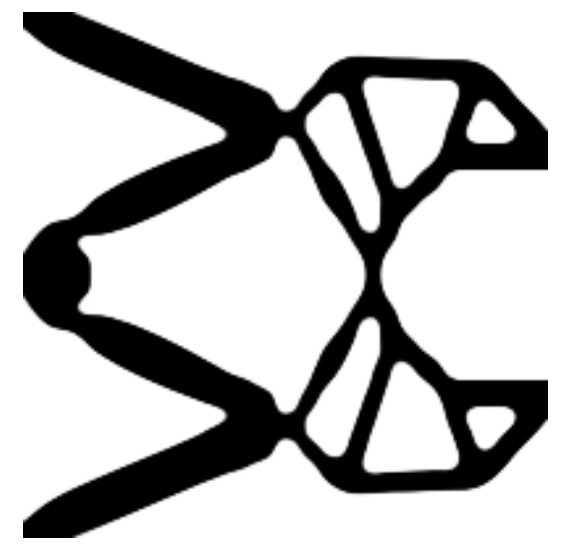

(c) Optimized designs obtained with a reanalysis procedure, 5 PCG iterations. $f_{0}=-1.8649, m_{f}=-1.8172, \sigma_{f}=$ $0.036, C I=(-1.8185,-1.8159)$

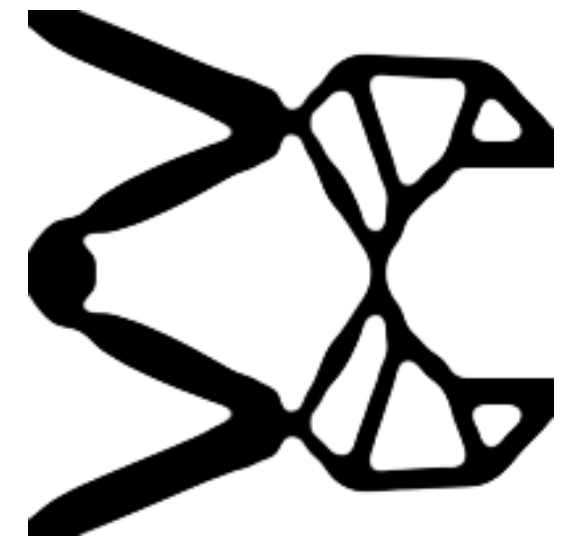

(b) Optimized designs obtained with a reanalysis procedure, 3 PCG iterations. $f_{0}=-1.8666, m_{f}=-1.8188, \sigma_{f}=$ $0.036, C I=(-1.8201,-1.8175)$

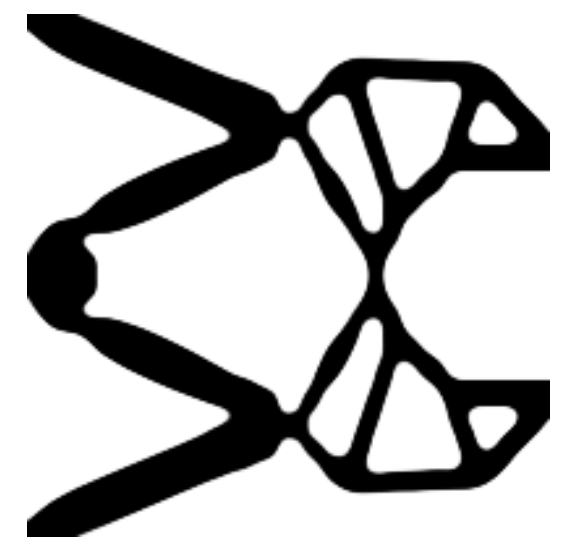

(d) Optimized designs obtained with a standard procedure. $f_{0}=-1.8656, m_{f}=-1.8174, \sigma_{f}=$ $0.036, C I=(-1.8187,-1.8161)$

Figure 11: Optimized layouts and performance of robust grippers obtained using reanalysis procedures with approximate sensitivity analysis. Confidence intervals $(C I)$ are computed for $95 \%$ probability. In the bottom right figure, the result of the standard procedure is presented for comparison. 


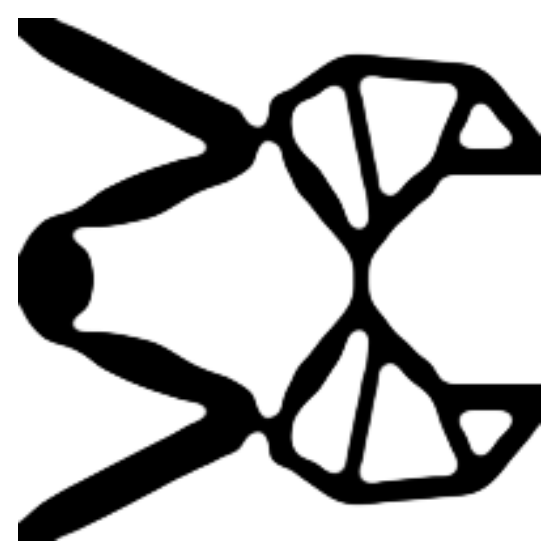

(a) Optimized designs obtained with a reanalysis procedure, 1 PCG iteration. $f_{0}=-1.8039, m_{f}=-1.7486, \sigma_{f}=$ $0.041, C I=(-1.7501,-1.7471)$

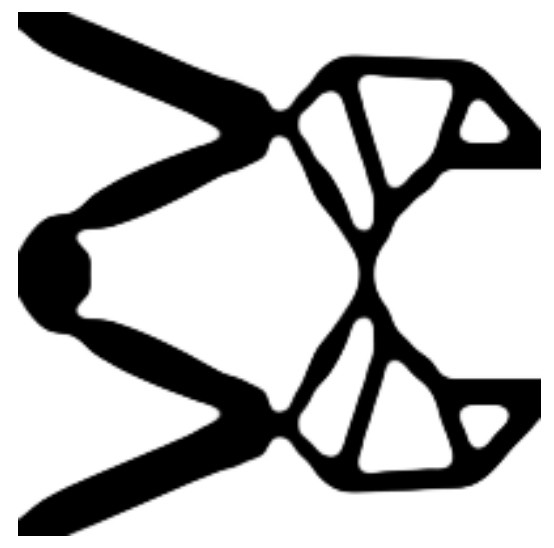

(c) Optimized designs obtained with a reanalysis procedure, 5 PCG iterations. $f_{0}=-1.8628, m_{f}=-1.8151, \sigma_{f}=$ $0.036, C I=(-1.8164,-1.8138)$

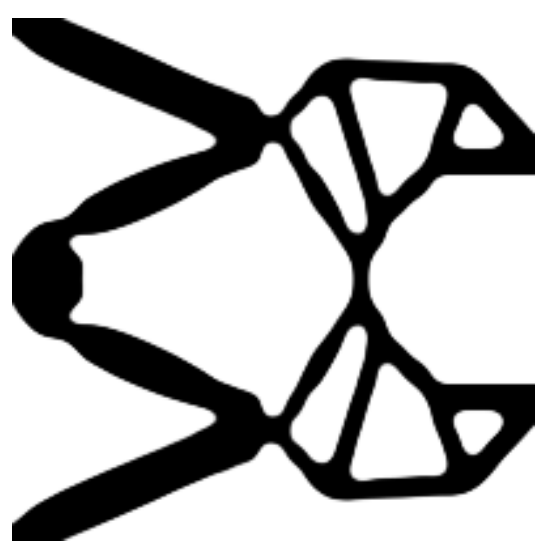

(b) Optimized designs obtained with a reanalysis procedure, 3 PCG iterations. $f_{0}=-1.8460, m_{f}=-1.8045, \sigma_{f}=$ $0.035, C I=(-1.8058,-1.8032)$

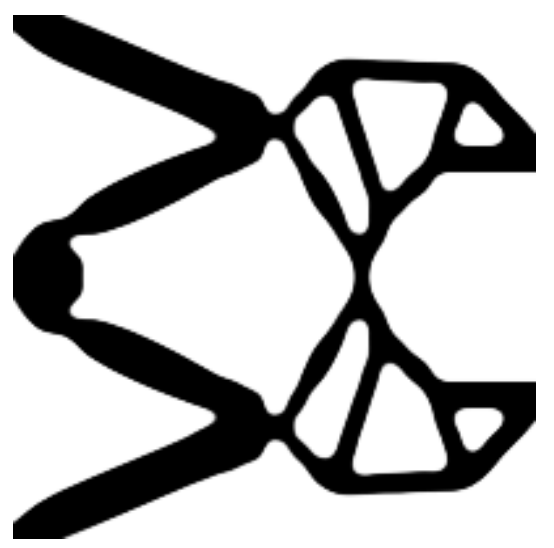

(d) Optimized designs obtained with a standard procedure. $f_{0}=-1.8656, m_{f}=-1.8174, \sigma_{f}=$ $0.036, C I=(-1.8187,-1.8161)$

Figure 12: Optimized layouts and performance of robust grippers obtained using reanalysis procedures with consistent sensitivity analysis. Confidence intervals $(C I)$ are computed for $95 \%$ probability. In the bottom right figure, the result of the standard procedure is presented for comparison.

The evaluated performance of the optimized grippers is presented in Table 5 . We focus on two quantities: $f_{0}$, the gripping performance of the blueprint design; and $m_{f}+\sigma_{f}$, the stochastic performance estimated using 3000 random realizations. As was the case in the previous example, variant 1 again appears to yield better results than variant 2 . However, when only a single PCG iteration is performed, the standard deviation corresponding to the design optimized with variant 1 is significantly higher than for all other designs. This is not the case when employing variant 2 where the standard deviation is similar for all the designs. Another advantage of using consistent sensitivity analysis is the monotonous convergence towards the result of the full procedure as the number of PCG iterations is increased, as can be observed also in Figure 13. 
Table 5: Performance of the gripper mechanism

\begin{tabular}{l|ccc|ccc} 
& \multicolumn{3}{|c|}{ Variant 1} & \multicolumn{3}{c}{ Variant 2} \\
\hline PCG iterations & $f_{0}$ & $m_{f}+\sigma_{f}$ & $\sigma_{f}$ & $f_{0}$ & $m_{f}+\sigma_{f}$ & $\sigma_{f}$ \\
\hline 1 & -1.8582 & -1.7180 & 0.0679 & -1.8039 & -1.7077 & 0.0409 \\
2 & -1.8668 & -1.7832 & 0.0355 & -1.8172 & -1.7397 & 0.0305 \\
3 & -1.8666 & -1.7832 & 0.0356 & -1.8460 & -1.7693 & 0.0352 \\
4 & -1.8681 & -1.7828 & 0.0360 & -1.8567 & -1.7770 & 0.0330 \\
5 & -1.8649 & -1.7817 & 0.0355 & -1.8628 & -1.7790 & 0.0361 \\
10 & -1.8604 & -1.7814 & 0.0344 & -1.8648 & -1.7822 & 0.0354 \\
\hline Direct solves & -1.8656 & -1.7814 & 0.0360 & -1.8656 & -1.7814 & 0.0360
\end{tabular}

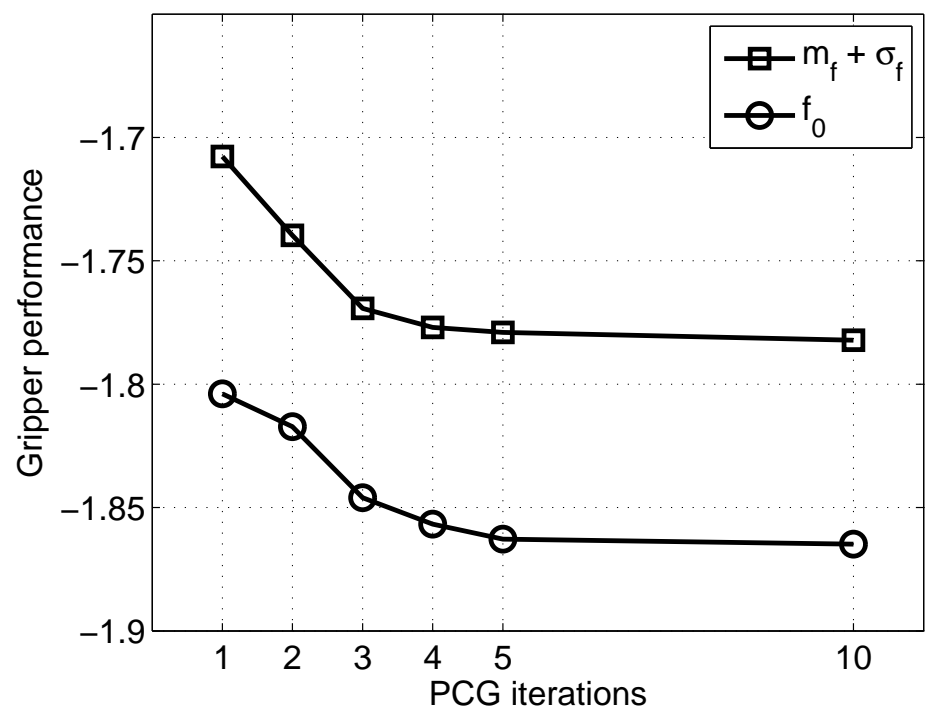

Figure 13: Actual performance of the optimized grippers for different accuracies of the reanalysis procedure with consistent sensitivity analysis.

\subsection{Example 3: re-using the reference design in consecutive design cycles}

An interesting extension of the presented approach is to combine it with reanalysis procedures that are based on re-using the factorization of the reference design in subsequent design cycles [Amir et al., 2009; Amir and Sigmund, 2011]. This means reanalysis is performed both in a "parallel' manner (multiple designs, same design cycle) as well as in a 'forward' manner (consecutive design cycles). A thorough examination of the prospects of such an approach is left for future work, but some promising results are given in the following.

We return to the worst-case design of a force inverter discussed in Section 4.1. The aim is to re-use factorizations corresponding to the dilated design while maintaining accuracy of the optimization process. A new factorization is performed if one of the following conditions is met: 1) First 10 design cycles; 2) The relative norm of the residual in the solution for the dilated structure exceeds $1 e-4$; 3) 10 consecutive design cycles were performed since the previous factorization. If at least one of the conditions is fulfilled, the stiffness matrix of the dilated structure is decomposed and reanalysis is performed for the other two structures. Otherwise, reanalysis is utilized to evaluate all three structures. The maximum number of PCG iterations was set to 5 , except for the first 10 cycles and for the cycles corresponding to the doubling of $\beta$ where 10 PCG iterations are allowed.

Implementing variant 1 based on approximate sensitivity analysis, the achieved objective 
value was -2.1192 while variant 2 with consistent sensitivities reached an objective of -2.0764 . These results correspond to relative differences of $1.2 \%$ and $3.2 \%$ respectively, compared to the objective achieved with full solves. The number of factorizations performed was 67 in the variant 1 experiment and 66 in the variant 2 experiment, compared to 900 in a standard approach and 300 in the reanalysis approach discussed throughout this article. Examining the optimized layouts, the goals of robust design are satisfied despite the minor difference in performance. In conclusion, this extended reanalysis approach is well worth exploring because further reduction in computational cost appears to be possible.

\subsection{Actual savings in computation}

The actual computational speedup expected to be achieved by implementing the proposed approach is estimated using the force inverter problem with various aspect ratios and corresponding FE mesh sizes. Executing a FORTRAN code, the running time of a single reanalysis was measured relatively to the running time of a standard FE direct solve. For this purpose variant 2 was implemented but the computational effort involved in variant 1 is roughly the same: the cost of performing a PCG iteration followed by a reverse adjoint-CG iteration is similar to the cost of performing a single block-PCG iteration. The relative computing times, measured in percentage of the time spent on a direct solve, alongside the achieved objective values, are presented in Table 6 . We note that the output performance depends on the aspect ratio so objectives should only be compared with other results obtained on the same FE mesh.

Table 6: Relative time for one reanalysis and objective values for various worst-case force inverter design problems

\begin{tabular}{l|cc|cc|cc|cc} 
FE mesh & \multicolumn{2}{|c|}{$400 \times 200$} & \multicolumn{2}{c|}{$400 \times 300$} & \multicolumn{2}{c|}{$400 \times 400$} & \multicolumn{2}{c}{$600 \times 300$} \\
\hline DOF & \multicolumn{2}{|c|}{161,202} & \multicolumn{2}{c|}{241,402} & \multicolumn{2}{c|}{321,602} & \multicolumn{2}{c}{361,802} \\
\hline & Time & Objective & Time & Objective & Time & Objective & Time & Objective \\
1 PCG iter. & $10.2 \%$ & -1.8596 & $8.8 \%$ & -1.8633 & $10.2 \%$ & -1.7172 & $7.7 \%$ & -1.7883 \\
2 PCG iter. & $18.1 \%$ & -1.9915 & $15.5 \%$ & -1.9091 & $12.9 \%$ & -1.7524 & $13.3 \%$ & -1.8930 \\
3 PCG iter. & $24.4 \%$ & -2.0046 & $21.5 \%$ & -1.8830 & $18.5 \%$ & -1.7516 & $21.4 \%$ & -1.9550 \\
4 PCG iter. & $31.8 \%$ & -2.0046 & $28.7 \%$ & -1.8819 & $25.9 \%$ & -1.7581 & $24.3 \%$ & -1.9494 \\
5 PCG iter. & $38.2 \%$ & -2.0126 & $34.8 \%$ & -1.8852 & $29.9 \%$ & -1.7599 & $29.5 \%$ & -1.9511 \\
10 PCG iter. & $65.7 \%$ & -2.0198 & $56.8 \%$ & -1.8858 & $48.9 \%$ & -1.7609 & $50.2 \%$ & -1.9632 \\
\hline Direct solve & $100.0 \%$ & -2.0300 & $100.0 \%$ & -1.8860 & $100.0 \%$ & -1.7590 & $100.0 \%$ & -1.9720
\end{tabular}

In the reanalysis schemes, the major cost is due to right-hand solves (2 per PCG iteration) and matrix-vector products (4 per PCG iteration if consistent sensitivities are derived). Denoting $n$ as the number of degrees of freedom; $b$ the bandwidth of the stiffness matrix; and $i$ the number of PCG iterations, the number of operations performed in reanalysis is proportional to $n \times b \times i$ whereas the cost of factorization is proportional to $n \times b^{2}$. This means that the relative cost of reanalysis reduces as the bandwidth increases. On the other hand, it is expected that the number of PCG iterations required to achieve sufficiently accurate reanalysis will increase with mesh refinement. For the four discretizations in Table 6, the theoretical relative times for one reanalysis with 10 PCG iterations are estimated as $40.2 \%, 26.7 \%, 20.0 \%$ and $26.7 \%$ respectively.

Some insight regarding the trade-off between the efficiency of reanalysis and the accuracy in the outcome of optimization is provided in Figure 14, which is based on the results from Table 6. In the experiments conducted, fairly accurate outcome (difference in performance smaller than 1\%) was achieved for the computational cost of around 30\% compared to a direct solve. We note that calculations based on operation counts lead to much more promising savings than those achieved in our experiments. For example, a single reanalysis on the $600 \times 300$ mesh with 10 PCG iterations is predicted to be more than 3 times faster than a direct solve, while a factor 
of only 2 was achieved in the experiment. This means that actual savings in larger problems depend not only on the properties of the problem but also on aspects of the implementation that are beyond the scope of the current study, such as: computer specifications; quality of programming; and availability of efficient scientific computing libraries.

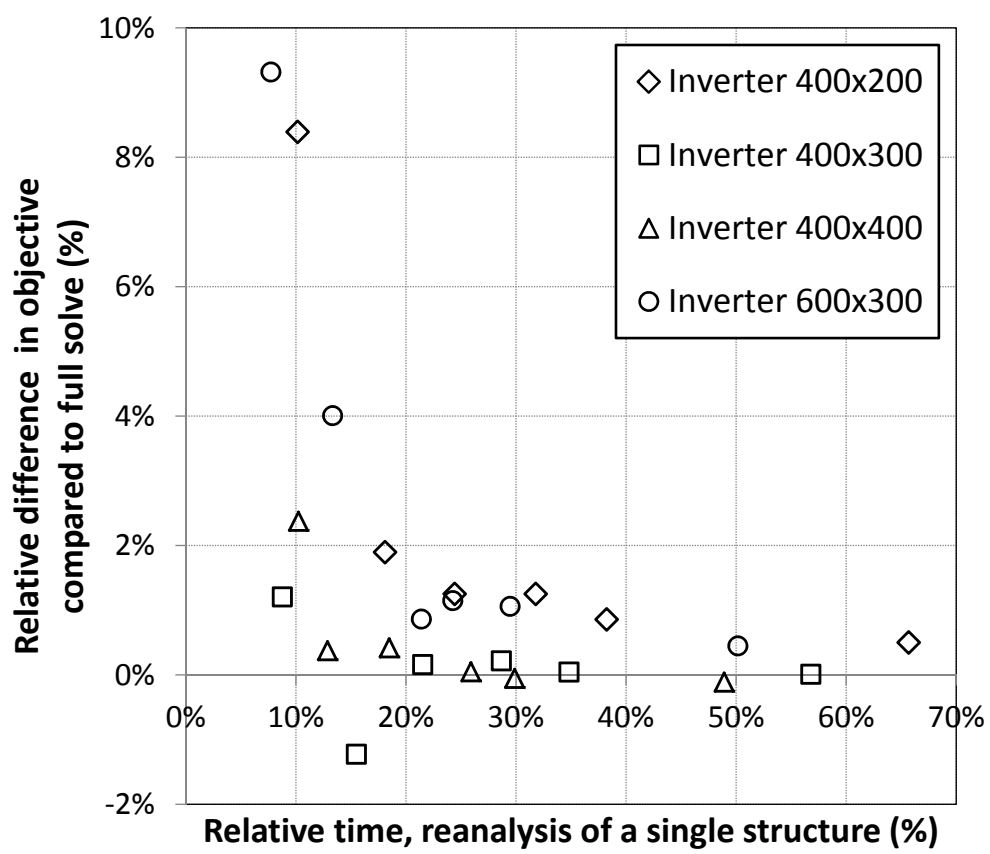

Figure 14: Trade-off between accuracy and efficiency based on numerical solution of various worst-case force inverter design problems.

Clearly, the benefit of applying reanalysis techniques increases with the number of structures to be evaluated within a single optimization cycle. For the purpose of this discussion, we assume a sufficiently accurate reanalysis takes $30 \%$ of the time needed for a direct solve. Then in the worst case approach with three designs, the time spent on solving the nested analysis problem will be roughly $53 \%$ of the time spent if three direct solves were performed. In the stochastic approach, distribution of the problem to parallel processors must be considered due to the large number of designs. Then the actual savings depend on the number of designs evaluated on a single processor. For example, if 1000 designs are evaluated and 100 processors are available, each processor will perform one factorization (of the reference design) followed by 10 reanalyses. This means the evaluation of 10 structures will take $40 \%$ of the time compared to 10 direct solves.

\section{Conclusions}

An efficient approach to robust topology optimization was presented. The high computational effort of solving multiple $\mathrm{FE}$ analyses is decreased by utilizing reanalysis procedures. Addressing two representative design problems, it was demonstrated that the benefits of applying a robust formulation can be achieved for a significantly reduced computational cost.

The reanalysis procedures applied in this study are based on performing a single stiffness matrix factorization per design cycle, corresponding to a 'reference' design. This factorization is then re-used in a reanalysis scheme to obtain approximations of the responses of multiple designs, the number of which may vary between a few to several hundreds or thousands, depending on the robust formulation employed. It was shown that using the uniformly dilated design as a reference design is a very effective choice, leading to a rapid reduction in the approximation errors in the first few reanalysis iterations. 
Two computational schemes were tested, involving either an approximate or a consistent sensitivity analysis. For the test cases considered, the approximate scheme denoted 'variant 1' appears to give more accurate results but this is not necessarily the case in general. Practical implementation of variant 1 is quite straightforward because it only requires a PCG (or preferably block-PCG) solver in addition to the code for robust topology optimization. Variant 2 is more consistent from a mathematical perspective but it requires the implementation of an adjoint sensitivity analysis for early termination of a PCG solver. Readers who are interested in further exploring this approach are welcome to contact the corresponding author for receiving a MATLAB or FORTRAN code containing the dedicated subroutine.

According to numerical experiments and predictions based on operation counts, the computational cost of multiple FE analyses can be reduced by at least $50 \%$ while maintaining sufficient accuracy. The extent of the actual savings depends on the properties of the problem in hand as well as on the efficiency of the computer code. Based on the results of the current study as well as on the conclusions of previous investigations, we believe that the key for deriving reanalysis procedures that yield sufficient accuracy for minimal computational cost lies in linking reanalysis and optimization. This means that ultimatively, the required accuracy of reanalysis will be defined rigorously within the optimization routine according to the progress of optimization.

A short glimpse into future work was given, where the exploitation of approximate reanalysis was extended to accommodate the use of a single matrix factorization within several design cycles. This was achieved by combining the present approach with previous developments regarding the integration of reanalysis into standard topology optimization procedures [Amir et al., 2009]. Another extension of the current study is to consider the case where a factorization cannot be stored due to memory limits. Then, reanalysis can be performed based on re-using a preconditioner that is related to the reference design and that requires less memory than the Cholesky factor.

\section{Acknowledgments}

The first author would like to thank Anton Evgrafov, Mathias Stolpe and Fengwen Wang for fruitful discussions on the topic. We thank the anonymous reviewers for their helpful comments on the manuscript. This work was financially supported by: The Danish Council for Independent Research | Technology and Production Sciences; Villum Fonden via the NextTop project; and the Danish Center of Scientific Computing (DCSC). These supports are gratefully acknowledged. The third author is a postdoctoral fellow of the Research Foundation - Flanders and a member of K.U.Leuven-BOF PFV/10/002 OPTEC - Optimization in Engineering Center. Finally, we thank the anonymous reviewers for their helpful comments and suggestions.

\section{References}

O. Amir and O. Sigmund. On reducing computational effort in topology optimization: how far can we go? Structural and Multidisciplinary Optimization, 44:25-29, 2011.

O. Amir, U. Kirsch, and I. Sheinman. Efficient non-linear reanalysis of skeletal structures using combined approximations. International Journal for Numerical Methods in Engineering, 73: 1328-1346, 2008.

O. Amir, M. P. Bendsøe, and O. Sigmund. Approximate reanalysis in topology optimization. International Journal for Numerical Methods in Engineering, 78:1474-1491, 2009.

O. Amir, M. Stolpe, and O. Sigmund. Efficient use of iterative solvers in nested topology optimization. Structural and Multidisciplinary Optimization, 42:55-72, 2010. 
M. P. Bendsøe and N. Kikuchi. Generating optimal topologies in structural design using a homogenization method. Computer Methods in Applied Mechanics and Engineering, 71:197$224,1988$.

M. P. Bendsøe and O. Sigmund. Topology Optimization - Theory, Methods and Applications. Springer, Berlin, 2003.

H.-G. Beyer and B. Sendhoff. Robust optimization - a comprehensive survey. Computer Methods in Applied Mechanics and Engineering, 196(33-34):3190 - 3218, 2007.

B. Bourdin. Filters in topology optimization. International Journal for Numerical Methods in Engineering, 50:2143-2158, 2001.

T. E. Bruns and D. A. Tortorelli. Topology optimization of non-linear elastic structures and compliant mechanisms. Computer Methods in Applied Mechanics and Engineering, 190:3443$3459,2001$.

K. Chen. Matrix Preconditioning Techniques and Applications. Cambridge University Press, Cambridge, 2005.

S. Chen and W. Chen. A new level-set based approach to shape and topology optimization under geometric uncertainty. Structural and Multidisciplinary Optimization, 44:1-18, 2011.

S. Chen, W. Chen, and S. Lee. Level set based robust shape and topology optimization under random field uncertainties. Structural and Multidisciplinary Optimization, 41:507-524, 2010.

J. Doob. Stochastic processes. John Wiley \& Sons, New York, USA, 1953.

R. Ghanem and P. Spanos. Stochastic finite elements: a spectral approach. Springer-Verlag, New York, 1991.

G. H. Golub and C. F. Van Loan. Matrix Computations. The Johns Hopkins University Press, Baltimore, Maryland, third edition, 1996.

J. K. Guest, J. H. Prévost, and T. Belytschko. Achieving minimum length scale in topology optimization using nodal design variables and projection functions. International Journal for Numerical Methods in Engineering, 61:238-254, 2004.

M. R. Hestenes and E. Stiefel. Methods of conjugate gradients for solving linear systems. Journal of Research of the National Bureau of Standards, 49(6):409-436, 1952.

U. Kirsch. Reduced basis approximations of structural displacements for optimal design. AIAA journal, 29:1751-1758, 1991.

U. Kirsch. Reanalysis of Structures. Springer, Dordrecht, 2008.

U. Kirsch and M. Bogomolni. Procedures for approximate eigenproblem reanalysis of structures. International Journal for Numerical Methods in Engineering, 60:1969-1986, 2004.

U. Kirsch and M. Bogomolni. Nonlinear dynamic reanalysis of structures by combined approximations. Computer Methods in Applied Mechanics and Engineering, 195:4420-4432, 2006.

U. Kirsch and P. Y. Papalambros. Structural reanalysis for topological modifications - a unified approach. Structural and Multidisciplinary Optimization, 21:333-344, 2001.

U. Kirsch, M. Kočvara, and J. Zowe. Accurate reanalysis of structures by a preconditioned conjugate gradient method. International Journal for Numerical Methods in Engineering, 55: 233-251, 2002. 
N. Kogiso, W. Ahn, S. Nishiwaki, K. Izui, and M. Yoshimura. Robust topology optimization for compliant mechanisms considering uncertainty of applied loads. Journal of Advanced Mechanical Design Systems and Manifacturing, 2(1):96-107, 2008.

A. Kolmogorov. Foundations of the theory of probability. Chelsea Publishing Company, New York, 2nd English edition, 1956.

B. S. Lazarov, M. Schevenels, and O. Sigmund. Robust design of large-displacement compliant mechanisms. Mechanical Sciences, 2(2):175-182, 2011. doi: 10.5194/ms-2-175-2011.

L. Leu and C. Huang. A reduced basis method for geometric nonlinear analysis of structures. journal of the International Association for Shell and Spatial Structures, 39:71-76, 1998.

C. Li and A. Der Kiureghian. Optimal discretization of random fields. ASCE Journal of Engineering Mechanics, 119(6):1136-1154, 1993.

J. Logo, M. Ghaemi, and M. Rad. Optimal topologies in case of probabilistic loading: The influence of load correlation. Mechanics Based Design of Structures and Machines, 37(3): 327-348, 2009.

D. P. O'Leary. The block conjugate gradient algorithm and related methods. Linear Algebra and its Applications, 29:293-322, 1980.

Y. Saad. Iterative Methods for Sparse Linear Systems, Second Edition. SIAM, 2003.

M. Schevenels, B. Lazarov, and O. Sigmund. Robust topology optimization accounting for spatially varying manufacturing errors. Computer Methods in Applied Mechanics and Engineering, 200(49-52):3613 - 3627, 2011. doi: 10.1016/j.cma.2011.08.006.

G. Schuller and H. Jensen. Computational methods in optimization considering uncertainties an overview. Computer Methods in Applied Mechanics and Engineering, 198(1):2-13, 2008.

O. Sigmund. Morphology-based black and white filters for topology optimization. Structural and Multidisciplinary Optimization, 33:401-424, 2007.

O. Sigmund. Manufacturing tolerant topology optimization. Acta Mechanica Sinica, 25:227-239, 2009 .

K. Svanberg. The method of moving asymptotes - a new method for structural optimization. International Journal for Numerical Methods in Engineering, 24:359-373, 1987.

M. Tootkaboni, A. Asadpoure, and J. K. Guest. Topology optimization of continuum structures under uncertainty - A polynomial chaos approach. Computer Methods in Applied Mechanics and Engineering, 201-204:263-275, 2012.

Y. Tsompanakis, N. D. Lagaros, and M. Papadrakakis, editors. Structural Design Optimization Considering Uncertainties, volume 1 of Structures $\&$ Infrastructures Series. Taylor \& Francis Group, 2008.

F. Wang, B. S. Lazarov, and O. Sigmund. On projection methods, convergence and robust formulations in topology optimization. Structural and Multidisciplinary Optimization, 43: 767-784, 2011.

W. Wilkinson. The Algebraic Eigenvalue Problem. Oxford University Press, Oxford, 1965.

S. Xu, Y. Cai, and G. Cheng. Volume preserving nonlinear density filter based on heaviside functions. Structural and Multidisciplinary Optimization, 41(4):495-505, APR 2010. doi: 10.1007/s00158-009-0452-7. 